\title{
SYSTEMATIC ENCODERS FOR GENERALIZED GABIDULIN CODES AND THE $q$-ANALOGUE OF CAUCHY MATRICES
}

\author{
ALESSANDRO NERI* \\ Technical University of Munich, Germany
}

\begin{abstract}
We characterize the generator matrix in standard form of generalized Gabidulin codes. The parametrization we get for the non-systematic part of this matrix coincides with the $q$-analogue of generalized Cauchy matrices, leading to the definition of $q$-Cauchy matrices. These matrices can be represented very conveniently and their representation allows to define new interesting subfamilies of generalized Gabidulin codes whose generator matrix is a structured matrix. In particular, as an application, we construct Gabidulin codes whose generator matrix is the concatenation of an identity block and a Toeplitz/Hankel matrix. In addition, our results allow to give a new efficient criterion to verify whether a rank metric code of dimension $k$ and length $n$ is a generalized Gabidulin code. This criterion is only based on the computation of the rank of one matrix and on the verification of the linear independence of two sets of elements and it requires $\mathcal{O}(m \cdot F(k, n))$ field operations, where $F(k, n)$ is the cost of computing the reduced row echelon form of a $k \times n$ matrix. Moreover, we also provide a characterization of the generator matrix in standard form of general MRD codes.
\end{abstract}

\section{INTRODUCTION}

Codes in the rank metric were introduced, independently, by Delsarte [13], Gabidulin [15] and Roth [37], although a similar notion can be traced back to Bergman [7]. However, only in the last ten years have they significantly gained interest, due to their application in network coding [45, 18]. Moreover, rank metric codes have a plethora of different applications in communications and security. In addition to network coding, the applications proposed in the last 20 years concern cryptography [22,34], space-time coding and wireless communications [47, 24], distributed storage [43, 9, 29], authentication schemes [32] and low-rank matrix completion [27].

As with codes in the Hamming metric, they are usually defined over a finite field $\mathbb{F}_{q}$, and in the linear case their important parameters are given by the length $n$, the dimension $k$ and the minimum distance $d$. Those parameters are related by an inequality that is as elegant as effective. This is the well-known Singleton bound, that holds in both the Hamming and rank metric. Hamming codes meeting this bound are called maximum distance separable (MDS) codes. Their natural analogue in the rank metric is represented by maximum rank distance $(M R D)$ codes, that are defined analogously as codes that attain the Singleton bound with equality. Although it was proven that there are plenty of MRD codes that are linear over the extension field [30, 8], only few new families have been discovered recently [41, 23, 33, 42, 35] and some sporadic construction [17, 10, 11, 12, 25, 5].

However, the most studied and important construction of MRD codes is still the one proposed in the seminal works [13, 15, 37], and then generalized in [20]. These codes are known

E-mail address: alessandro.neri@tum.de.

2010 Mathematics Subject Classification. 94B05; $11 \mathrm{~T} 71$.

Key words and phrases. Rank-metric codes; Gabidulin codes; $q$-Cauchy matrices; systematic Gabidulin codes; standard form.

*Alessandro Neri was supported by the Swiss National Science Foundation through grants no. 169510 and 187711 
as generalized Gabidulin codes, and they represent the rank analogue of the well-known generalized Reed-Solomon (GRS) codes. As GRS codes, generalized Gabidulin codes are evaluation codes. However, they are defined over an extension field $\mathbb{F}_{q^{m}}$ of $\mathbb{F}_{q}$ and the evaluation is done on a particular subset of linearized polynomials in $n$ points that are linearly independent over $\mathbb{F}_{q}$. The structure of evaluation codes allowed the development of many efficient decoding algorithms in the last years [44, 48].

In this framework, another analogy emerges regarding the generator matrices of these two families of codes. The canonical generator matrix of GRS codes is obtained by the evaluation of the canonical basis $\left\{1, x, \ldots, x^{k-1}\right\}$, that gives as a result the weighted Vandermonde matrix, a matrix given by the product of a Vandermonde with a non-singular diagonal matrix. The rank analogue of the weighted Vandermonde matrix is given by the $s$-Moore matrix. Such a matrix is the canonical generator matrix of a generalized Gabidulin code, obtained via the evaluation of the canonical basis $\left\{x, x^{q^{s}}, x^{q^{2 s}}, \ldots, x^{q^{(k-1) s}}\right\}$.

There is another important generator matrix of GRS codes that is well-known in the literature. In 1985 Roth and Seroussi gave a characterization of the generator matrix in standard form for these codes, showing that GRS codes are in correspondence with generalized Cauchy matrices ([40]). The same characterization was also given independently by Dür in [14]. Explicitly, the generator matrix in standard form of a GRS code is given by $\left(I_{k} \mid X\right)$, where $I_{k}$ is the $k \times k$ identity matrix, and $X$ is a generalized Cauchy matrix. On the other hand, every matrix $\left(I_{k} \mid X\right)$, with $X$ a generalized Cauchy matrix, generates a GRS code.

In this work we give a characterization of the generator matrix in standard form of a generalized Gabidulin code, that up to now was unknown. As a consequence, this also allows us to define a rank analogue of generalized Cauchy matrices, whose definition coincides with the $q$-analogue of generalized Cauchy matrices. This result is obtained making a wide use of properties of finite fields, in particular the trace map, and of some recent results appeared recently $[17,30]$.

In addition to the theoretical result that almost completes the picture on the analogies between GRS and generalized Gabidulin codes, this has also a useful impact from a practical point of view. Using the structure of the rank analogue of a generalized Cauchy matrix, we derive a subfamily of generalized Gabidulin codes whose generator matrix is made by an identity block and a Toeplitz/Hankel block. From an application point of view, this new family of codes seems to be suitable for fast algorithms for erasure correction and syndrome decoding as well as for encoding. It is well-known, indeed, that the matrix-vector multiplication with a Toeplitz/Hankel matrix can be performed in a fast way.

Moreover, from the theoretical characterization obtained, we also derive a new criterion to determine whether a given code is a generalized Gabidulin code. This new criterion is faster to compute than any other previously known. Indeed, for a given rank metric code of dimension $k$ and length $n$ over a finite field $\mathbb{F}_{q^{m}}$, it only requires $\mathcal{O}(m \cdot F(k, n))$ field operations, where $F(k, n)$ denotes the cost of computing the reduced row echeleon form of a $k \times n$ matrix.

The paper is structured as follows. In Section 2 we recall some basic properties of finite fields and in particular of the field trace map. We also briefly explain the main results on GRS codes and on their generator matrices. In Section 3 we introduce rank metric codes and give a recap on the most important results on MRD and generalized Gabidulin codes. In addition, some new results are presented that are preparatory for the rest of the paper. Moreover, we give a characterization of the generator matrix of general MRD codes, in the spirit of the wellknown results for MDS codes. Section 4 represents the main contribution of this work. Here we characterize the generator matrix in standard form of a generalized Gabidulin code. From this result we derive a new criterion for determining if a given rank metric code is a generalized Gabidulin code. This section can be also seen as the analogue of Roth and Seroussi [40] and Dür [14] works for the rank metric and it completes the picture on the generator matrices of generalized Gabidulin codes. In Section 5 we use our previous results for constructing subfamilies of Gabidulin codes with structured generator matrix. These codes have generator matrix in standard form with a Hankel or a Toeplitz non-systematic part, potentially very 
useful for applications. Finally, in Section 6 we summarize the work underlining our main contributions.

\section{PReliminaries}

Throughout the whole work, given a map $h: \mathcal{X} \rightarrow \mathcal{Y}$ and a subset $\mathcal{T} \subseteq \mathcal{Y}$, we denote by $h^{-1}(\mathcal{T})$ the preimage of the set $\mathcal{T}$, i.e.

$$
h^{-1}(\mathcal{T})=\{x \in \mathcal{X} \mid h(x) \in \mathcal{T}\} .
$$

In the same way, for a set $\mathcal{S} \subseteq \mathcal{X}, h(\mathcal{S})$ denotes the set of images of the elements in $\mathcal{S}$ through $h$, i.e.

$$
h(\mathcal{S})=\{h(x) \mid x \in \mathcal{S}\} .
$$

2.1. Trace over finite fields an its duality. The following definitions and results can be found in any textbook on finite fields, e.g. [21]. We denote the finite field of cardinality $q$ by $\mathbb{F}_{q}$. It is well-known that it exists if and only if $q$ is a prime power. Moreover, if it exists, $\mathbb{F}_{q}$ is unique up to isomorphism. An extension field of extension degree $m$ is denoted by $\mathbb{F}_{q^{m}}$. An important property of finite fields is the existence of a primitive element. This means that there always exists $\alpha \in \mathbb{F}_{q}$ that is a generator of $\mathbb{F}_{q}^{*}$, i.e.

$$
\mathbb{F}_{q}=\{0\} \cup\left\{\alpha^{i} \mid 0 \leq i \leq q-2\right\} .
$$

We now recall some basic theory on finite fields and the trace function. It is well-known that the extension field $\mathbb{F}_{q^{m}}$ over $\mathbb{F}_{q}$ is a Galois extension and $\operatorname{Gal}\left(\mathbb{F}_{q^{m}} / \mathbb{F}_{q}\right)$ is cyclic. One of its generators is given by the $q$-Frobenius automorphism $\theta$, defined as

$$
\begin{aligned}
\theta: \mathbb{F}_{q^{m}} & \longrightarrow \mathbb{F}_{q^{m}} \\
\alpha & \longmapsto \alpha^{q} .
\end{aligned}
$$

Definition 1. Let $\mathbb{F}_{q}$ be a finite field and $\mathbb{F}_{q^{m}}$ be an extension field. For $\alpha \in \mathbb{F}_{q^{m}}$, the trace of $\alpha$ over $\mathbb{F}_{q}$ is defined by

$$
\operatorname{Tr}_{\mathbb{F}_{q^{m}} / \mathbb{F}_{q}}(\alpha):=\sum_{i=0}^{m-1} \theta^{i}(\alpha)=\sum_{i=0}^{m-1} \alpha^{q^{i}} .
$$

For every integer $0<s<m$ with $\operatorname{gcd}(m, s)=1$, we denote by $\varphi_{s}$ the map given by

$$
\begin{aligned}
\varphi_{s}: \mathbb{F}_{q^{m}} & \longrightarrow \mathbb{F}_{q^{m}} \\
\alpha & \longmapsto \theta^{s}(\alpha)-\alpha .
\end{aligned}
$$

We will refer to the function

$$
\operatorname{Tr}_{\mathbb{F}_{q} / \mathbb{F}_{q}}: \mathbb{F}_{q^{m}} \longrightarrow \mathbb{F}_{q}
$$

as the trace map of $\mathbb{F}_{q^{m}} / \mathbb{F}_{q}$.

The following result relates the trace map with the functions $\varphi_{s}$.

Lemma 1. The trace map satisfies the following properties:

(1) $\operatorname{Tr}_{\mathbb{F}_{q} m / \mathbb{F}_{q}}(\alpha) \in \mathbb{F}_{q}$ for all $\alpha \in \mathbb{F}_{q^{m}}$.

(2) $\operatorname{Tr}_{\mathbb{F}_{q} / \mathbb{F}_{q}}$ is an $\mathbb{F}_{q}$-linear surjective transformation from $\mathbb{F}_{q^{m}}$ to $\mathbb{F}_{q}$.

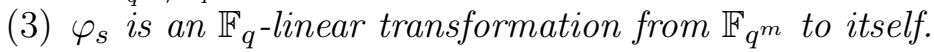

(4) For every s coprime to $m, \varphi_{s}(\alpha)=0$ if and only if $\alpha \in \mathbb{F}_{q}$.

(5) (Additive Hilbert's Theorem 90 for finite fields) $\operatorname{ker}\left(\operatorname{Tr}_{\mathbb{F}_{q} m / \mathbb{F}_{q}}\right)=\operatorname{Im}\left(\varphi_{s}\right)$ for every $s$ coprime to $m$ and has cardinality $q^{m-1}$.

Proof. A partial proof of this result can be found in [21, Chapter 2, Section 3]. For a complete proof we refer to [30, Lemma 2].

The trace map has many important properties. One of them is that it can be used to define an isomorphism between $\mathbb{F}_{q^{m}}$ and $\operatorname{Hom}_{\mathbb{F}_{q}}\left(\mathbb{F}_{q^{m}}, \mathbb{F}_{q}\right)$. 
Definition 2. The $\mathbb{F}_{q}$-bilinear map defined as

is called the trace form of the extension $\mathbb{F}_{q^{m}} / \mathbb{F}_{q}$.

$$
\begin{aligned}
\operatorname{tr}: \mathbb{F}_{q^{m}} \times \mathbb{F}_{q^{m}} & \longrightarrow \mathbb{F}_{q} \\
(\alpha, \beta) & \longmapsto \operatorname{Tr}_{\mathbb{F}_{q} / \mathbb{F}_{q}}(\alpha \beta),
\end{aligned}
$$

Observe that for every $\alpha \in \mathbb{F}_{q^{m}}$, we can associate an $\mathbb{F}_{q^{-}}$linear map $T_{\alpha} \in \operatorname{Hom}_{\mathbb{F}_{q}}\left(\mathbb{F}_{q^{m}}, \mathbb{F}_{q}\right)$, defined as

$$
\begin{aligned}
T_{\alpha}: \mathbb{F}_{q^{m}} & \longrightarrow \mathbb{F}_{q} \\
\beta & \longmapsto \operatorname{Tr}_{\mathbb{F}_{q^{m} / \mathbb{F}_{q}}}(\alpha \beta) .
\end{aligned}
$$

Theorem 1. The trace form is a symmetric non degenerate $\mathbb{F}_{q}$-bilinear form. Moreover it induces a duality isomorphism given by

$$
\begin{aligned}
\Psi: \mathbb{F}_{q^{m}} & \longrightarrow \operatorname{Hom}_{\mathbb{F}_{q}}\left(\mathbb{F}_{q^{m}}, \mathbb{F}_{q}\right) \\
\alpha & \longmapsto T_{\alpha} .
\end{aligned}
$$

Proof. For the proof one can see [21, Theorem 2.24].

The following results directly follow from Theorem 1 .

Corollary 1. For every $\alpha \in \mathbb{F}_{q^{m}}^{*}$ the map $T_{\alpha}$ is non identically zero, and hence $\operatorname{dim}_{\mathbb{F}_{q}}\left(\operatorname{ker}\left(T_{\alpha}\right)\right)=$ $m-1$.

Corollary 2. For every $\alpha, \beta \in \mathbb{F}_{q^{m}}$ and $\lambda, \mu \in \mathbb{F}_{q}$, we have

$$
T_{\lambda \alpha+\mu \beta}=\lambda T_{\alpha}+\mu T_{\beta} .
$$

Since the trace form induces a duality isomorphism, we can naturally define the notion of dual basis.

Definition 3. Given an $\mathbb{F}_{q^{\text {-basis }}} \alpha_{1}, \ldots, \alpha_{m}$ of $\mathbb{F}_{q^{m}}$ and $\beta_{1}, \ldots, \beta_{m} \in \mathbb{F}_{q^{m}}$, we say that $\beta_{1}, \ldots, \beta_{m}$ is a dual basis of $\alpha_{1}, \ldots, \alpha_{m}$ with respect to the trace form, if for all $i, j \in\{1, \ldots, m\}$

$$
\operatorname{tr}\left(\alpha_{i}, \beta_{j}\right)=\delta_{i, j}= \begin{cases}1 & \text { if } i=j \\ 0 & \text { if } i \neq j .\end{cases}
$$

Remark 1. Given an $\mathbb{F}_{q^{\text {-basis }}} \alpha_{1}, \ldots, \alpha_{m}$ of $\mathbb{F}_{q^{m}}$, the existence and uniqueness of its dual basis follow by Theorem 1 and the fact that $\mathbb{F}_{q^{m}}$ is a finite dimensional $\mathbb{F}_{q^{-}}$-vector space.

Lemma 2. For every $\alpha_{1}, \ldots, \alpha_{k}, \beta \in \mathbb{F}_{q^{m}}$,

$$
\operatorname{ker}\left(T_{\alpha_{1}}\right) \cap \ldots \cap \operatorname{ker}\left(T_{\alpha_{k}}\right) \subseteq \operatorname{ker}\left(T_{\beta}\right)
$$

if and only if $\beta \in\left\langle\alpha_{1}, \ldots, \alpha_{k}\right\rangle$.

Proof. Suppose $\beta \in\left\langle\alpha_{1}, \ldots, \alpha_{k}\right\rangle$. By Corollary 2, we have $T_{\beta}=\lambda_{1} T_{\alpha_{1}}+\ldots+\lambda_{k} T_{\alpha_{k}}$. Hence, if $x \in \operatorname{ker}\left(T_{\alpha_{1}}\right) \cap \ldots \cap \operatorname{ker}\left(T_{\alpha_{k}}\right)$, then

$$
T_{\beta}(x)=\lambda_{1} T_{\alpha_{1}}(x)+\ldots+\lambda_{k} T_{\alpha_{k}}(x)=0+\ldots+0=0,
$$

and therefore $x \in \operatorname{ker}\left(T_{\beta}\right)$.

On the other hand, suppose $\beta \notin\left\langle\alpha_{1}, \ldots, \alpha_{k}\right\rangle$. Let $s:=\operatorname{dim}_{\mathbb{F}_{q}}\left\langle\alpha_{1}, \ldots, \alpha_{k}\right\rangle$. Without loss of generality we can assume that $\left\langle\alpha_{1}, \ldots, \alpha_{k}\right\rangle=\left\langle\alpha_{1}, \ldots, \alpha_{s}\right\rangle$. Now, complete $\alpha_{1}, \ldots, \alpha_{s}, \beta$

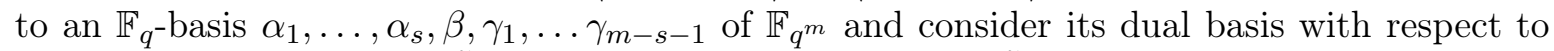
the trace form $\tilde{\alpha}_{1}, \ldots, \tilde{\alpha}_{s}, \tilde{\beta}, \tilde{\gamma}_{1}, \ldots \tilde{\gamma}_{m-s-1}$. Therefore, $T_{\alpha_{i}}(\tilde{\beta})=0$ for every $i=1, \ldots, s$ and $T_{\beta}(\tilde{\beta})=1$, i.e.

$$
\tilde{\beta} \in \operatorname{ker}\left(T_{\alpha_{1}}\right) \cap \ldots \cap \operatorname{ker}\left(T_{\alpha_{k}}\right) \backslash \operatorname{ker}\left(T_{\beta}\right) .
$$

Proposition 1. For every $\alpha_{1}, \ldots, \alpha_{k} \in \mathbb{F}_{q^{m}}$,

$$
\operatorname{dim}_{\mathbb{F}_{q}}\left(\operatorname{ker}\left(T_{\alpha_{1}}\right) \cap \ldots \cap \operatorname{ker}\left(T_{\alpha_{k}}\right)\right)=m-\operatorname{dim}_{\mathbb{F}_{q}}\left\langle\alpha_{1}, \ldots, \alpha_{k}\right\rangle .
$$


Proof. Let $s:=\operatorname{dim}_{\mathbb{F}_{q}}\left\langle\alpha_{1}, \ldots, \alpha_{k}\right\rangle$. Without loss of generality we can suppose $\left\langle\alpha_{1}, \ldots, \alpha_{k}\right\rangle=$ $\left\langle\alpha_{1}, \ldots, \alpha_{s}\right\rangle$. By Lemma 2 we have $\operatorname{ker}\left(T_{\alpha_{s+1}}\right), \ldots, \operatorname{ker}\left(T_{\alpha_{k}}\right) \supseteq \operatorname{ker}\left(T_{\alpha_{1}}\right) \cap \ldots \cap \operatorname{ker}\left(T_{\alpha_{s}}\right)$, and hence

$$
\operatorname{ker}\left(T_{\alpha_{1}}\right) \cap \ldots \cap \operatorname{ker}\left(T_{\alpha_{k}}\right)=\operatorname{ker}\left(T_{\alpha_{1}}\right) \cap \ldots \cap \operatorname{ker}\left(T_{\alpha_{s}}\right) .
$$

Therefore, it is enough to prove the statement when $\alpha_{1}, \ldots, \alpha_{k}$ are linearly independent over $\mathbb{F}_{q}$. We use induction on $k$. If $k=1$ then $\operatorname{dim}_{\mathbb{F}_{q}}\left(\operatorname{ker}\left(T_{\alpha_{1}}\right)\right)=m-1$ by Corollary 1 .

Suppose now that the statement is true for $k-1$, i.e.

$$
\operatorname{dim}_{\mathbb{F}_{q}}(S)=m-k+1
$$

where $S:=\operatorname{ker}\left(T_{\alpha_{1}}\right) \cap \ldots \cap \operatorname{ker}\left(T_{\alpha_{k-1}}\right)$. Then, by Lemma $2, S \nsubseteq \operatorname{ker}\left(T_{\alpha_{k}}\right)$, i.e. $S+\operatorname{ker}\left(T_{\alpha_{k}}\right)=$ $\mathbb{F}_{q^{m}}$. Therefore,

$$
\begin{aligned}
\operatorname{dim}_{\mathbb{F}_{q}}\left(S \cap \operatorname{ker}\left(T_{\alpha_{k}}\right)\right) & =\operatorname{dim}_{\mathbb{F}_{q}}(S)+\operatorname{dim}_{\mathbb{F}_{q}}\left(\operatorname{ker}\left(T_{\alpha_{k}}\right)\right)-\operatorname{dim}_{\mathbb{F}_{q}}\left(S+\operatorname{ker}\left(T_{\alpha_{k}}\right)\right) \\
& =m-k+1+m-1-m \\
& =m-k .
\end{aligned}
$$

Now, let $\alpha_{1}, \ldots, \alpha_{k} \in \mathbb{F}_{q^{m}}$ be $\mathbb{F}_{q^{-}}$-linearly independent and complete them to a basis $\alpha_{1}, \ldots, \alpha_{m}$ of $\mathbb{F}_{q^{m}}$. Let $\beta_{1}, \ldots, \beta_{m}$ be its dual bases. Then for every $i=1, \ldots, k$ we have $T_{\alpha_{i}}\left(\beta_{j}\right)=0$ for every $j=k+1, \ldots, m$, i.e. $\beta_{j} \in \operatorname{ker}\left(T_{\alpha_{1}}\right) \cap \ldots \cap \operatorname{ker}\left(T_{\alpha_{k}}\right)$. Moreover, by Proposition 1 , we get $\operatorname{dim}_{\mathbb{F}_{q}}\left(\operatorname{ker}\left(T_{\alpha_{1}}\right) \cap \ldots \cap \operatorname{ker}\left(T_{\alpha_{k}}\right)\right)=m-k$, and hence

$$
\operatorname{ker}\left(T_{\alpha_{1}}\right) \cap \ldots \cap \operatorname{ker}\left(T_{\alpha_{k}}\right)=\left\langle\beta_{k+1}, \ldots, \beta_{m}\right\rangle .
$$

We can now define the trace-orthogonal space of a subspace as follows.

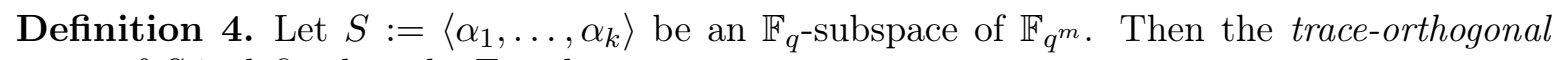
space of $S$ is defined as the $\mathbb{F}_{q}$-subspace

$$
S^{\times}:=\operatorname{ker}\left(T_{\alpha_{1}}\right) \cap \ldots \cap \operatorname{ker}\left(T_{\alpha_{k}}\right) .
$$

Proposition 2. The subspace $S^{\times}$is well-defined, i.e. it does not depend on the choice of the set of generators.

Proof. Let $\left\{\alpha_{1}, \ldots, \alpha_{k}\right\}$ and $\left\{\alpha_{1}^{\prime}, \ldots, \alpha_{t}^{\prime}\right\}$ be two sets of generators for a subspace $S$. We want to prove that $\operatorname{ker}\left(T_{\alpha_{1}}\right) \cap \ldots \cap \operatorname{ker}\left(T_{\alpha_{k}}\right)=\operatorname{ker}\left(T_{\alpha_{1}^{\prime}}\right) \cap \ldots \cap \operatorname{ker}\left(T_{\alpha_{t}^{\prime}}\right)$. For every $i=1, \ldots, k$, $\alpha_{i} \in\left\langle\alpha_{1}^{\prime}, \ldots, \alpha_{t}^{\prime}\right\rangle$ and therefore, by Lemma 2 , it holds that $\operatorname{ker}\left(T_{\alpha_{i}}\right) \supseteq \operatorname{ker}\left(T_{\alpha_{1}^{\prime}}\right) \cap \ldots \cap \operatorname{ker}\left(T_{\alpha_{t}^{\prime}}\right)$. Hence,

$$
\operatorname{ker}\left(T_{\alpha_{1}}\right) \cap \ldots \cap \operatorname{ker}\left(T_{\alpha_{k}}\right) \supseteq \operatorname{ker}\left(T_{\alpha_{1}^{\prime}}\right) \cap \ldots \cap \operatorname{ker}\left(T_{\alpha_{t}^{\prime}}\right) .
$$

The opposite inclusion is analogous.

We already know the relation between the image of the map $\varphi_{s}$ and the kernel of the trace map (see Lemma 1). The following Lemma characterizes the preimage of any element under the $\operatorname{map} \varphi_{s}$.

Lemma 3. Let $\alpha \in \mathbb{F}_{q^{m}}$ and $s$ a positive integer coprime to $m$. Then

$$
\left|\varphi_{s}^{-1}(\{\alpha\})\right|= \begin{cases}q & \text { if } \alpha \in \operatorname{ker}\left(\operatorname{Tr}_{\mathbb{F}_{q} / \mathbb{F}_{q}}\right) \\ 0 & \text { if } \alpha \notin \operatorname{ker}\left(\operatorname{Tr}_{\mathbb{F}_{q} m / \mathbb{F}_{q}}\right)\end{cases}
$$

(2) Let $\alpha \in \operatorname{ker}\left(\operatorname{Tr}_{\mathbb{F}_{q} m / \mathbb{F}_{q}}\right)$. If $x_{1}, x_{2} \in \varphi_{s}^{-1}(\{\alpha\})$, then $x_{1}-x_{2} \in \mathbb{F}_{q}$, or equivalently, there exists an $x \in \mathbb{F}_{q^{m}}$ such that

$$
\varphi_{s}^{-1}(\{\alpha\})=\left\{x+\lambda \mid \lambda \in \mathbb{F}_{q}\right\} .
$$

Moreover such an $x$ is of the form 


$$
x=-\frac{1}{\operatorname{Tr}_{\mathbb{F}_{q} m / \mathbb{F}_{q}}(\gamma)} \sum_{i=0}^{m-2}\left(\sigma^{i+1}(\gamma) \sum_{j=0}^{i}\left(\sigma^{j}(\alpha)\right)\right) .
$$

where $\gamma \in \mathbb{F}_{q^{m}}$ is such that $\operatorname{Tr}_{\mathbb{F}_{q^{m}} / \mathbb{F}_{q}}(\gamma) \neq 0$, and $\sigma:=\theta^{s}$.

Proof. (1) If $\alpha \notin \operatorname{ker}\left(\operatorname{Tr}_{\mathbb{F}_{q} m / \mathbb{F}_{q}}\right)$, then, by part (5) of Lemma 1 we have $\varphi_{s}^{-1}(\{\alpha\})=\emptyset$. On the other hand, if $\alpha \in \operatorname{ker}\left(\operatorname{Tr}_{\mathbb{F}_{q} m / \mathbb{F}_{q}}\right)$, then $\left|\varphi_{s}^{-1}(\{\alpha\})\right|=\left|\operatorname{ker}\left(\varphi_{s}\right)\right|$, since $\varphi_{s}$ is an $\mathbb{F}_{q}$-linear map. By part (2) of Lemma 1

$$
q^{m-1}=\left|\operatorname{ker}\left(\operatorname{Tr}_{\mathbb{F}_{q} / \mathbb{F}_{q}}\right)\right|=\left|\operatorname{Im}\left(\varphi_{s}\right)\right|=\frac{\left|\mathbb{F}_{q^{m}}\right|}{\left|\operatorname{ker}\left(\varphi_{s}\right)\right|}
$$

and therefore we get $\left|\varphi_{s}^{-1}(\{\alpha\})\right|=q$.

(2) For the first part, let $x_{1}, x_{2} \in \varphi_{s}^{-1}(\{\alpha\})$. Hence, $\varphi_{s}\left(x_{1}\right)-\varphi_{s}\left(x_{2}\right)=0$, and by linearity of $\varphi_{s}$, we get $\varphi_{s}\left(x_{1}-x_{2}\right)=0$. By part (4) of Lemma 1 we get $x_{1}-x_{2} \in \mathbb{F}_{q}$. Finally, showing that $\varphi_{s}(x)=\alpha$ is a straightforward computation.

We conclude this section with a useful result on the linear independence of preimages of $\varphi_{s}$.

Lemma 4. Let $\alpha_{1}, \ldots, \alpha_{k} \in \operatorname{ker}\left(\operatorname{Tr}_{\mathbb{F}_{q} m / \mathbb{F}_{q}}\right), s$ be a positive integer coprime to $m$ and $\sigma:=\theta^{s}$. Suppose moreover that $\beta_{1}, \ldots, \beta_{k} \in \mathbb{F}_{q^{m}}$ are such that $\sigma\left(\beta_{i}\right)-\beta_{i}=\alpha_{i}$. Then, the elements $\alpha_{1}, \ldots, \alpha_{k}$ are linearly independent over $\mathbb{F}_{q}$ if and only if the elements $1, \beta_{1}, \ldots, \beta_{k}$ are linearly independent over $\mathbb{F}_{q}$.

Proof. Suppose $\lambda_{1}, \ldots, \lambda_{k} \in \mathbb{F}_{q}$ and consider the sum

$$
\sum_{i} \lambda_{i} \alpha_{i}=\sum_{i} \lambda_{i}\left(\sigma\left(\beta_{i}\right)-\beta_{i}\right)=\sigma\left(\sum_{i} \lambda_{i} \beta_{i}\right)-\sum_{i} \lambda_{i} \beta_{i}
$$

This means that a non-trivial combination of the $\alpha_{i}$ 's is zero if and only if a non-trivial combination of the $\beta_{i}$ 's belongs to $\operatorname{ker} \varphi_{s}$. This is equivalent, by part (4) of Lemma 1, to $\sum_{i} \lambda_{i} \beta_{i} \in \mathbb{F}_{q}$, i.e. $1, \beta_{1}, \ldots, \beta_{k}$ are linearly dependent over $\mathbb{F}_{q}$.

2.2. GRS codes and Generalized Cauchy Matrices. In classical coding theory the most studied and well-known class of codes is definitely represented by generalized Reed-Solomon codes. These codes were introduced in [36] and through the years were deeply studied by many authors. Their importance is due to the fact that they are maximum distance separable, and possess very fast algorithms for their encoding and decoding procedures [16, 19]. In this section we are going to briefly describe them, focusing in particular on their generator matrices.

Let $n$ be a positive integer. The Hamming distance $d_{H}$ on $\mathbb{F}_{q}^{n}$ is defined as

$$
\begin{aligned}
d_{H}: \mathbb{F}_{q}^{n} \times \mathbb{F}_{q}^{n}: & \longrightarrow \mathbb{N} \\
(u, v) & \longmapsto\left|\left\{i \mid u_{i} \neq v_{i}\right\}\right| .
\end{aligned}
$$

It is well-known that $d_{H}$ defines indeed a metric on $\mathbb{F}_{q}^{n}$. With this metric, classical coding theory was developed in the last 70 years, focusing on many different classes of codes. In this section we will only consider linear codes.

Definition 5. Let $0<k \leq n$ be two positive integers. A linear code $\mathcal{C}$ of dimension $k$ and length $n$ over a finite field $\mathbb{F}_{q}$ is a $k$-dimensional $\mathbb{F}_{q}$-subspace of $\mathbb{F}_{q}^{n}$ equipped with the Hamming distance. The minimum distance of $\mathcal{C}$ is the integer

$$
d_{H}(\mathcal{C}):=\min \left\{d_{H}(u, v) \mid u, v \in \mathcal{C}, u \neq v\right\} .
$$

A matrix $G \in \mathbb{F}_{q}^{k \times n}$ is called a generator matrix for the code $\mathcal{C}$ if $\mathcal{C}=\operatorname{rs}(G)$, where $\operatorname{rs}(G)$ denotes the subspace generated by the rows of $G$, called the row space of $G$. 
It is well known that the minimum distance $d$ of any linear code of dimension $k$ and length $n$ satisfies the following inequality:

$$
d \leq n-k+1
$$

This bound is known as Singleton bound [46] and codes meeting it with equality are called called maximum distance separable (MDS) codes.

Among all the possible generator matrices of an MDS code, there exists one in a special form. Indeed, it is easy to verify that every MDS code of length $n$ and dimension $k$ has a generator matrix of the form $G=\left(I_{k} \mid X\right)$, where $X \in \mathbb{F}_{q}^{k \times(n-k)}$ and $I_{k}$ denotes the $k \times k$ identity matrix. Such a generator matrix is said to be in standard form, or equivalently, in systematic form. Hence, for a given matrix $X \in \mathbb{F}_{q}^{k \times(n-k)}$, we denote by $\mathcal{C}_{X}$ the code generated by $\left(I_{k} \mid X\right)$. It is well-known that MDS codes can be characterized by the non-systematic part of their generator matrix in standard form. Concretely, we have the following result.

Theorem 2. A linear code $\mathcal{C}_{X} \subseteq \mathbb{F}_{q}^{n}$ is $M D S$ if and only if the matrix $X$ is superregular ${ }^{1}$

Let $0<k \leq n$ be two positive integers, and consider the set of polynomials over $\mathbb{F}_{q}$ of degree strictly less than $k$

$$
\mathbb{F}_{q}[x]_{<k}:=\left\{f(x) \in \mathbb{F}_{q}[x] \mid \operatorname{deg} f<k\right\} .
$$

Definition 6. Suppose moreover that $n \leq q$, and consider $\alpha_{1}, \ldots, \alpha_{n} \in \mathbb{F}_{q}$ pairwise distinct elements, and $b_{1}, \ldots, b_{n} \in \mathbb{F}_{q}^{*}$. The code

$$
\mathcal{C}=\left\{\left(b_{1} f\left(\alpha_{1}\right), b_{2} f\left(\alpha_{2}\right), \ldots, b_{n} f\left(\alpha_{n}\right)\right) \mid f \in \mathbb{F}_{q}[x]_{<k}\right\}
$$

is called Generalized Reed-Solomon (GRS) code and it is denoted by $\operatorname{GRS}_{n, k}(\alpha, b)$, where $\alpha=\left(\alpha_{1}, \ldots, \alpha_{n}\right)$ and $b=\left(b_{1}, \ldots, b_{n}\right)$.

It is well-known that GRS codes are MDS and that the canonical generator matrix for a GRS code $\mathcal{C}=\operatorname{GRS}_{n, k}(\alpha, b)$ is given by the weighted Vandermonde matrix that is

$$
\left(\begin{array}{cccc}
b_{1} & b_{2} & \ldots & b_{n} \\
b_{1} \alpha_{1} & b_{2} \alpha_{2} & \ldots & b_{n} \alpha_{n} \\
b_{1} \alpha_{1}^{2} & b_{2} \alpha_{2}^{2} & \ldots & b_{n} \alpha_{n}^{2} \\
\vdots & \vdots & & \vdots \\
b_{1} \alpha_{1}^{k-1} & b_{2} \alpha_{2}^{k-1} & \ldots & b_{n} \alpha_{n}^{k-1}
\end{array}\right)=V_{k}(\alpha) \operatorname{diag}(b)
$$

where $V_{k}(\alpha)$ is the classical Vandermonde matrix, and $\operatorname{diag}(b)$ denotes the diagonal matrix whose diagonal entries are given by $b_{1}, \ldots, b_{n}$. This generator matrix is obtained by choosing the set of monomials $\left\{1, x, x^{2}, \ldots, x^{k-1}\right\}$ as an $\mathbb{F}_{q}$-basis of $\mathbb{F}_{q}[x]_{<k}$, and then evaluating each of them in the points $\alpha_{1}, \ldots, \alpha_{n}$. This is why we refer to it as the canonical generator matrix.

In 1985 Roth and Seroussi [40] studied the generator matrix in standard form of a GRS code, giving a complete characterization. The same result was given by Dür in [14].

Definition 7. Let $r, s$ be positive integers, $x_{1}, \ldots, x_{r}, y_{1}, \ldots, y_{s} \in \mathbb{F}_{q}$, and $c_{1}, \ldots, c_{r}, d_{1}, \ldots, d_{s} \in$ $\mathbb{F}_{q}^{*}$ be elements such that

(a) $x_{1}, \ldots, x_{r}$ pairwise distinct,

(b) $y_{1}, \ldots, y_{s}$ pairwise distinct,

(c) $y_{i} \in \mathbb{F}_{q} \backslash\left\{x_{1}, \ldots, x_{r}\right\}$, for $i=1, \ldots, s$.

The matrix $C \in \mathbb{F}_{q}^{r \times s}$ defined by

is called Generalized Cauchy (GC) matrix.

$$
C_{i, j}=\frac{c_{i} d_{j}}{x_{i}-y_{j}}
$$

Theorem 3. [40, Theorem 1], [14, Theorem 2]

(1) If $\mathcal{C}=\operatorname{GRS}_{n, k}(\alpha, b)$, then $\mathcal{C}=\mathcal{C}_{X}$, where $X \in \mathbb{F}_{q}^{k \times(n-k)}$ is a GC matrix.

\footnotetext{
${ }^{1} \mathrm{~A}$ matrix $A \in \mathbb{F}^{r \times t}$ is said to be superregular if all its minors are nonzero
} 
(2) If $X \in \mathbb{F}_{q}^{k \times(n-k)}$ is a GC matrix then the code $\mathcal{C}_{X}$ is a Generalized Reed-Solomon code.

Theorem 3 gives a correspondence between GRS codes of dimension $k$ and length $n$ over $\mathbb{F}_{q}$, and $k \times(n-k)$ GC matrices over $\mathbb{F}_{q}$. Moreover, in [39, Lemma 7], a characterization of the GC in terms of its entries was given. We are now going to reformulate this result for our purpose, in order to underline that it gives a way to determine whether a code is a GRS code in terms of its generator matrix in standard form.

Let $A \in\left(\mathbb{F}_{q}^{*}\right)^{r \times s}$ with entries $a_{i, j}$. We denote by $A^{(-1)}$ the $r \times s$ matrix over $\mathbb{F}_{q}^{*}$ whose entries are $a_{i, j}^{-1}$.

Theorem 4. [39] Let $X \in \mathbb{F}_{q}^{k \times(n-k)}$ Then, the code $\mathcal{C}_{X}$ is a GRS code if and only if

(i) every entry $x_{i, j}$ is non-zero,

(ii) every $2 \times 2$ minor of $X^{(-1)}$ is non-zero, and

(iii) $\operatorname{rk}\left(X^{(-1)}\right)=2$.

In the following we will see that the analogue of GRS in the rank metric is given by generalized Gabidulin codes. We will find the same kind of correspondence between them and the rank analogue of GC matrices, obtained by characterizing their generator matrix in standard form. Moreover, we will also find an analogue of Theorem 4 in that framework.

\section{RAnK Metric Codes}

In this section we will give a recap about rank metric codes. In particular, we will only study those that linear over the extension field. Given a finite field $\mathbb{F}_{q}$ and an extension field

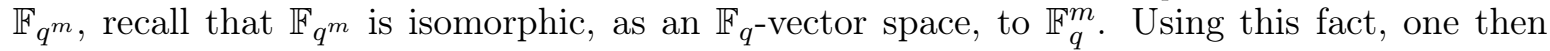
easily obtains the isomorphic description of matrices over the base field $\mathbb{F}_{q}$ as vectors over the extension field, i.e. $\mathbb{F}_{q}^{m \times n} \cong \mathbb{F}_{q^{m}}^{n}$. In this setting, let $g=\left(g_{1}, \ldots, g_{n}\right) \in \mathbb{F}_{q^{m}}^{n}$. We define the $\mathbb{F}_{q^{m} \text {-support of } g \text { over }} \mathbb{F}_{q}$ the $\mathbb{F}_{q^{-} \text {-subspace }}$

$$
\operatorname{supp}_{q}(g):=\left\langle g_{1}, \ldots, g_{n}\right\rangle_{\mathbb{F}_{q}} .
$$

Moreover, we denote by $\operatorname{rk}_{q}(g)=\operatorname{dim}_{\mathbb{F}_{q}}\left(\operatorname{supp}_{q}(g)\right)$, which is called the $q$-rank of $g$.

Unless otherwise specified, whenever we talk about vectors in $\mathbb{F}^{n}$ over a field $\mathbb{F}$, in this work we will always mean row vectors.

Definition 8. The rank distance $d_{R}$ on $\mathbb{F}_{q}^{m \times n}$ is defined by

$$
\mathrm{d}_{R}(X, Y):=\operatorname{rk}(X-Y), \quad X, Y \in \mathbb{F}_{q}^{m \times n} .
$$

Analogously, we define the rank distance between two elements $\boldsymbol{x}, \boldsymbol{y} \in \mathbb{F}_{q^{m}}^{n}$ as

$$
\mathrm{d}_{r}(x, y):=\operatorname{rk}_{q}(x-y),
$$

which corresponds to the rank of the difference of the respective matrix representations in $\mathbb{F}_{q}^{m \times n}$.

In this paper we will focus on $\mathbb{F}_{q^{m}}$-linear rank metric codes in $\mathbb{F}_{q^{m}}^{n}$, i.e. those codes that form a subspace of $\mathbb{F}_{q^{m}}^{n}$.

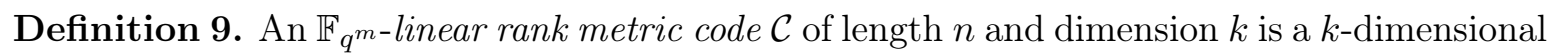
$\mathbb{F}_{q^{m}}$-subspace of $\mathbb{F}_{q^{m}}^{n}$ equipped with the rank distance $\mathrm{d}_{r}$.

As in the Hamming metric case, one defines the minimum rank distance of $\mathcal{C}$ as

$$
\mathrm{d}_{r}(\mathcal{C}):=\min \left\{\mathrm{d}_{r}(u, v) \mid u, v \in \mathcal{C}, u \neq v\right\},
$$

and a generator matrix $G \in \mathbb{F}_{q^{m}}^{k \times n}$ as a matrix whose row space (over $\mathbb{F}_{q^{m}}$ ) is $\mathcal{C}$.

The well-known Singleton bound for codes in the Hamming metric implies an upper bound for rank metric codes. 
Theorem 5. [15, Section 2] Let $\mathcal{C} \subseteq \mathbb{F}_{q^{m}}^{n}$ be an $\mathbb{F}_{q^{m} \text {-linear rank metric code with minimum }}$ rank distance $d$ of dimension $k$. Then

$$
d \leq n-k+1
$$

Definition 10. A rank metric code meeting the bound in Theorem 5 is called a maximum rank distance (MRD) code.

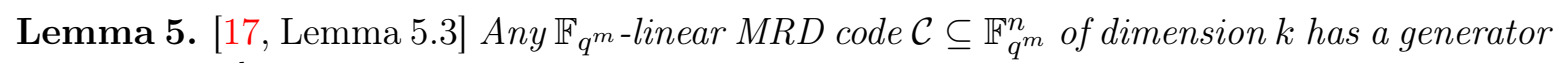
matrix $G \in \mathbb{F}_{q^{m}}^{k \times n}$ of the form

Moreover, all entries in $X$ are from $\mathbb{F}_{q^{m}} \backslash \mathbb{F}_{q}$.

$$
G=\left(I_{k} \mid X\right) \text {. }
$$

A generator matrix of the form $G=\left(I_{k} \mid X\right)$ is said to be in standard form (also called systematic form). The matrix $X$ of this representation is the non-systematic part of $G$.

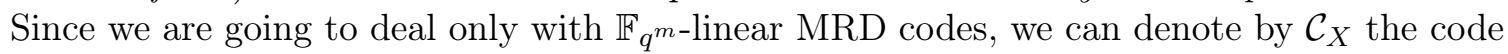
generated by $\left(I_{k} \mid X\right)$. In fact, by Lemma 5 every MRD code can be represented in a unique way as a code of the form $\mathcal{C}_{X}$ for some $X \in \mathbb{F}_{q^{m}}^{k \times(n-k)}$. We will widely use this notation later in this work.

It can be easily shown that a necessary condition for the existence of MRD codes is $n \leq m$. Therefore, in the rest of the paper we will always consider positive integers $k, n, m$ such that $0<k<n \leq m$.

Furthermore, the condition $n \leq m$ is also sufficient. In [13, 15] a general construction for MRD codes is given, which has been then generalized in [20]. In order to present such a construction we need to introduce a particular class of polynomials.

Definition 11. A linearized polynomial over $\mathbb{F}_{q^{m}}$ is a polynomial $f(x) \in \mathbb{F}_{q^{m}}[x] /\left(x^{q^{m}}-x\right)$ of the form

$$
\sum_{i=0}^{m-1} f_{i} x^{[i]}
$$

where $[i]:=q^{i}$. We denote by $\mathcal{L}_{m}\left(\mathbb{F}_{q^{m}}\right)$ the space of linearized polynomials over $\mathbb{F}_{q^{m}}$.

Let $\mathcal{G}_{k, s} \subseteq \mathcal{L}_{m}\left(\mathbb{F}_{q^{m}}\right)$ be the set defined as

$$
\mathcal{G}_{k, s}:=\left\{f_{0} x+f_{1} x^{[s]}+\ldots+f_{k-1} x^{[s(k-1)]} \mid f_{i} \in \mathbb{F}_{q^{m}}\right\} .
$$

Definition 12. Let $g=\left(g_{1}, \ldots, g_{n}\right) \in \mathbb{F}_{q^{m}}^{n}$ be a vector with $\operatorname{rk}_{q}(g)=n$ and let $s$ be an integer coprime to $m$. Let $\mathcal{C}$ be the rank metric code defined as

$$
\mathcal{C}=\left\{\left(f\left(g_{1}\right), f\left(g_{2}\right), \ldots, f\left(g_{n}\right)\right) \mid f \in \mathcal{G}_{k, s}\right\} .
$$

Then $\mathcal{C}$ is called generalized Gabidulin code of parameter $s$, and it will be denoted by

$$
\mathcal{C}=\mathcal{G}_{k, s}(g)
$$

We denote by $\mathrm{GL}_{n}(q):=\left\{A \in \mathbb{F}_{q}^{n \times n} \mid \operatorname{rk}(A)=n\right\}$ the general linear group of degree $n$ over $\mathbb{F}_{q}$. Furthermore, given a finite field $\mathbb{F}_{q}$, we consider the Grassmannian $\operatorname{Gr}\left(k, \mathbb{F}_{q}^{n}\right)$, that is the set of all $k$-dimensional subspaces of the vector space $\mathbb{F}_{q}^{n}$ over $\mathbb{F}_{q}$. It is well known that its cardinality is given by the Gaussian binomial $\left(\begin{array}{l}n \\ k\end{array}\right)_{q}$, defined as

$$
\left(\begin{array}{l}
n \\
k
\end{array}\right)_{q}=\prod_{i=0}^{k-1} \frac{q^{n}-q^{i}}{q^{k}-q^{i}}=\frac{\prod_{i=0}^{k-1}\left(q^{n}-q^{i}\right)}{\left|\mathrm{GL}_{k}(q)\right|}
$$

With this notation, for a positive integer $s$ coprime to $m$, we introduce the set $\operatorname{Gab}_{q}(k, n, m, s)$ as the set of all generalized Gabidulin codes over $\mathbb{F}_{q^{m}}$ of dimension $k$, length $n$ and parameter $s$, i.e.

$$
\operatorname{Gab}_{q}(k, n, m, s):=\left\{\mathcal{U} \in \operatorname{Gr}\left(k, \mathbb{F}_{q^{m}}^{n}\right) \mid \mathcal{U} \text { is a gen. Gabidulin code of parameter } s\right\} .
$$


Definition 13. For a vector $v:=\left(v_{1}, \ldots, v_{n}\right) \in \mathbb{F}_{q^{m}}^{n}$ we denote the $k \times n s$-Moore matrix by

$$
M_{s, k}(v):=\left(\begin{array}{cccc}
v_{1} & v_{2} & \ldots & v_{n} \\
\theta^{s}\left(v_{1}\right) & \theta^{s}\left(v_{2}\right) & \ldots & \theta^{s}\left(v_{n}\right) \\
\vdots & & & \vdots \\
\theta^{(k-1) s}\left(v_{1}\right) & \theta^{(k-1) s}\left(v_{2}\right) & \ldots & \theta^{(k-1) s}\left(v_{n}\right)
\end{array}\right) .
$$

At this point, it is straightforward to see that a generator matrix of a generalized Gabidulin code $\mathcal{G}_{k, s}(g)$ is given by the $k \times n s$-Moore matrix $M_{s, k}(g)$. This generator matrix is said to be canonical, since it is obtained by evaluating the basis of monomials $\left\{x, x^{[s]}, x^{[2 s]}, \ldots, x^{[(k-1) s]}\right\}$ of $\mathcal{G}_{k, s}$ in the points $g_{1}, \ldots, g_{n}$. Therefore, the $s$-Moore matrix is the natural rank analogue of a weighted Vandermonde matrix.

Note that for $s=1$, Definition 12 coincides with the classical Gabidulin code construction. The following theorem was shown for $s=1$ in [15, Section 4], and for general $s$ in [20].

Theorem 6. Let $1 \leq k \leq n \leq m$ be integers and let $s$ be another integer coprime to $m$. Moreover, let $g \in \mathbb{F}_{q^{m}}^{n}$ be such that $\operatorname{rk}_{q}(g)=n$. Then, the generalized Gabidulin code $\mathcal{G}_{k, s}(g) \subseteq$ $\mathbb{F}_{q^{m}}^{n}$ of dimension $k$ over $\mathbb{F}_{q^{m}}$ has minimum rank distance $n-k+1$. Thus, generalized Gabidulin codes are $\mathbb{F}_{q^{m} \text {-linear } M R D \text { codes. }}$

The dual code of a code $\mathcal{C} \subseteq \mathbb{F}_{q^{m}}^{n}$ is defined in the usual way as

$$
\mathcal{C}^{\perp}:=\left\{\boldsymbol{u} \in \mathbb{F}_{q^{m}}^{n} \mid \boldsymbol{u} \boldsymbol{c}^{\top}=0 \quad \forall \boldsymbol{c} \in \mathcal{C}\right\}
$$

In his seminal paper Gabidulin showed the following two results on dual codes of MRD and Gabidulin codes. The result was generalized to $s>1$ later on by Kshevetskiy and Gabidulin. Observe that also Delsarte in [13] proved a similar result for what concerns the dual of matrix codes with respect to the Delsarte bilinear form.

Proposition 3. [15, Sections 2 and 4][20, Subsection IV.C]

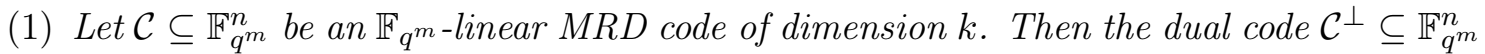

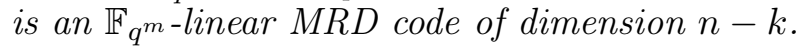

(2) Let $\mathcal{C} \subseteq \mathbb{F}_{q^{m}}^{n}$ be a generalized Gabidulin code of dimension $k$ and parameter $s$. Then the dual code $\mathcal{C}^{\perp} \subseteq \mathbb{F}_{q^{m}}^{n}$ is a generalized Gabidulin code of dimension $n-k$ and parameter $s$.

Given a matrix (resp. a vector) $A \in \mathbb{F}_{q^{m}}^{k \times n}$, we denote by $\theta^{s}(A)$ the component-wise $q$ Frobenius of $A$ applied $s$ times, i.e. $\theta^{s}(A)$ is generated by applying $\theta^{s}$ to every entry of the matrix (resp. the vector) $A$. Analogously, given a code $\mathcal{C} \subseteq \mathbb{F}_{q^{m}}^{k \times n}$, we define

$$
\theta^{s}(\mathcal{C}):=\left\{\theta^{s}(\mathbf{c}) \mid \mathbf{c} \in \mathcal{C}\right\} .
$$

Moreover we consider the map $\Phi_{s}$ defined as

$$
\begin{aligned}
\Phi_{s}: \mathbb{F}_{q^{m}}^{k \times(n-k)} & \longrightarrow \mathbb{F}_{q^{m}}^{k \times(n-k)} \\
X & \longmapsto \theta^{s}(X)-X .
\end{aligned}
$$

Observe that $\Phi_{s}$ is the function that maps every entry $x_{i, j}$ of the matrix $X$ to $\varphi_{s}\left(x_{i, j}\right)$.

Here we present some criteria on the generator matrix of a rank metric code, that allow to verify whether the code is MRD or generalized Gabidulin. We will need these results later on. The following criterion was given in [30, Proposition 22], and it improves [17, Corollary 2.12], which in turn is based on a well-known result given in [15]. First we define the sets

$$
\begin{aligned}
& \mathcal{E}_{q}(k, n):=\left\{E \in \mathbb{F}_{q}^{k \times n} \mid \operatorname{rk}_{q}(E)=k\right\} \\
& \mathcal{T}_{q}(k, n):=\left\{E \in \mathcal{E}_{q}(k, n) \mid E \text { is in reduced row echelon form }\right\}
\end{aligned}
$$


Proposition 4 (new MRD criterion). [30, Proposition 22] Let $G \in \mathbb{F}_{q^{m}}^{k \times n}$ be a generator matrix of a rank metric code $\mathcal{C} \subseteq \mathbb{F}_{q^{m}}^{n}$. Then $\mathcal{C}$ is an MRD code if and only if

$$
\operatorname{rk}\left(E G^{\top}\right)=k
$$

for all $E \in \mathcal{T}_{q}(k, n)$.

Furthermore, we need the following criterion for generalized Gabidulin codes.

Theorem 7 (gen. Gabidulin criterion). [30, Lemma 19] Let $X \in \mathbb{F}_{q^{m}}^{k \times(n-k)}$ such that $\mathcal{C}_{X} \subseteq \mathbb{F}_{q^{m}}^{n}$

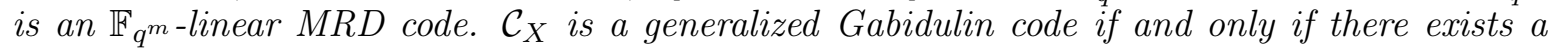
positive integer $s$ with $\operatorname{gcd}(s, m)=1$, such that

$$
\operatorname{rk}\left(\Phi_{s}(X)\right)=1
$$

Theorem 7 will be one of the most important results on which this work is based. The criterion starts with the assumption that we already know that the code is MRD. However, in Section 4 we will derive a new criterion that does not have such assumption and it is definitely easier to verify.

Concerning Gabidulin codes, we can also find the exact number of them. In [6], Berger provided the following result.

Proposition 5. [6, Theorem 2] Let $0<k<n$, and let $g=\left(g_{1}, \ldots, g_{n}\right), g^{\prime}=\left(g_{1}^{\prime}, \ldots, g_{n}^{\prime}\right) \in$ $\mathbb{F}_{q^{m}}^{n}$ be two vectors such that $\operatorname{rk}_{q}(g)=\operatorname{rk}_{q}\left(g^{\prime}\right)=n$. Then, for any integer $s$ coprime to $m$, $\operatorname{rs}\left(M_{s, k}(g)\right)=\operatorname{rs}\left(M_{s, k}\left(g^{\prime}\right)\right)$ if and only if $g=\lambda g^{\prime}$ for some $\lambda \in \mathbb{F}_{q^{m}}^{*}$.

Corollary 3. The number of k-dimensional generalized Gabidulin codes of length $n$ and $p a-$ rameter $s$ over $\mathbb{F}_{q^{m}}$ satisfies

$$
\left|\operatorname{Gab}_{q}(k, n, m, s)\right|=\prod_{i=1}^{n-1}\left(q^{m}-q^{i}\right) .
$$

Denote by $\operatorname{Aut}\left(\mathbb{F}_{q^{m}}\right)$ the automorphism group of $\mathbb{F}_{q^{m}}$. It is well-known that, if $q=p^{h}$ for a prime $p$, then $\operatorname{Aut}\left(\mathbb{F}_{q^{m}}\right)$ is generated by the Frobenius map, which takes an element to its $p$-th power. Hence, the automorphisms are of the form $x \mapsto x^{p^{i}}$ for some $0 \leq i<h m$.

The semilinear rank isometries on $\mathbb{F}_{q^{m}}^{n}$ are induced by the isometries on $\mathbb{F}_{q}^{m \times n}$ and are hence well-known, see e.g. [6, 26, 49].

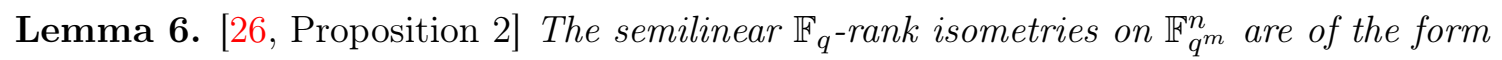

$$
(\lambda, A, \sigma) \in\left(\mathbb{F}_{q^{m}}^{*} \times \mathrm{GL}_{n}(q)\right) \rtimes \operatorname{Aut}\left(\mathbb{F}_{q^{m}}\right),
$$

acting on $\mathbb{F}_{q^{m}}^{n}$ via

$$
\left(v_{1}, \ldots, v_{n}\right) \cdot(\lambda, A, \sigma)=\left(\sigma\left(\lambda v_{1}\right), \ldots, \sigma\left(\lambda v_{n}\right)\right) A .
$$

In particular, if $\mathcal{C} \subseteq \mathbb{F}_{q^{m}}^{n}$ is a linear code with minimum rank distance $d$, then $\mathcal{C}^{\prime}:=\sigma(\lambda \mathcal{C}) A$ is a linear code with minimum rank distance $d$.

As semilinear isometries on $\mathbb{F}_{q^{m}}^{n}$ preserve the rank, we get that $\mathbb{F}_{q^{-}}$-linearly independent elements in $\mathbb{F}_{q^{m}}^{n}$ remain $\mathbb{F}_{q^{-}}$-linearly independent under the actions of $\left(\mathbb{F}_{q^{m}}^{*} \times \mathrm{GL}_{n}(q)\right) \rtimes \operatorname{Aut}\left(\mathbb{F}_{q^{m}}\right)$. Moreover, the $s$-Moore matrix structure is preserved under these actions, which implies that the class of generalized Gabidulin codes is closed under the semilinear isometries. Thus, a code is semilinearly isometric to a generalized Gabidulin code if and only if it is itself a generalized Gabidulin code.

As a consequence of Lemma 6, we have an interesting result, that will be useful in the next section.

Corollary 4. Let $X \in \mathbb{F}_{q^{m}}^{k \times(n-k)}$, and $\tilde{X}=X+B$ for some matrix $B \in \mathbb{F}_{q}^{k \times(n-k)}$. Moreover, let $s$ be a positive integer coprime to $m$.

(1) If the code $\mathcal{C}_{X}$ is $M R D$, then also $\mathcal{C}_{\tilde{X}}$ is $M R D$. 
(2) If the code $\mathcal{C}_{X}$ is a generalized Gabidulin code of parameter $s$, then also $\mathcal{C}_{\tilde{X}}$ is a generalized Gabidulin code of parameter s.

Proof. (1) Let $G=\left(I_{k} \mid X\right)$, be the generator matrix in standard form for $\mathcal{C}_{X}$, and let $\widetilde{G}=\left(I_{k} \mid \tilde{X}\right)$. Then, $\widetilde{G}=G M$ where

$$
M=\left(\begin{array}{cc}
I_{k} & B \\
0 & I_{n-k}
\end{array}\right) \in \mathrm{GL}_{n}(q) .
$$

By Lemma $6, \mathcal{C}_{\tilde{X}}=\mathcal{C}_{X} M$ is MRD.

(2) By Theorem 6 the code $C_{X}$ is MRD, and so it is $C_{\tilde{X}}$ by part (1) of this Corollary. Moreover we have

$$
\Phi_{s}(\tilde{X})=\Phi_{s}(X+B)=\Phi_{s}(X)
$$

and we conclude using Theorem 7 .

We now give an easy improvement of Lemma 5.

Lemma 7. Let $X=\left(x_{i, j}\right) \in \mathbb{F}_{q^{m}}^{k \times(n-k)}$.

(1) If there exists $i$ such that $\operatorname{rk}_{q}\left(1, x_{i, 1}, \ldots, x_{i, n-k}\right)<n-k+1$, then $\mathcal{C}_{X}$ is not MRD.

(2) If there exists $j$ such that $\operatorname{rk}_{q}\left(1, x_{1, j}, \ldots, x_{k, j}\right)<k+1$, then $\mathcal{C}_{X}$ is not MRD.

Proof. (1) Suppose that $1, x_{i, 1}, \ldots, x_{i, n-k}$ are $\mathbb{F}_{q}$-linearly dependent for some $i \in\{1, \ldots, k\}$, and consider the non-zero codeword

$$
e_{i}\left(I_{k} \mid X\right)=\left(0, \ldots, 0,1,0, \ldots, 0, x_{i, 1}, \ldots, x_{i, n-k}\right) .
$$

The rank of this codeword is strictly less than $n-k+1$, and therefore $\mathcal{C}_{X}$ can not be MRD.

(2) In this case we consider the code $\mathcal{C}_{X}^{\frac{1}{X}}$. Since a generator matrix for this code is $\left(-X^{\top} \mid\right.$ $I_{n-k}$ ), we get that $\mathcal{C}_{X}^{\perp}$ is permutation equivalent to the code $\mathcal{C}_{-X^{\top}}$. By the first part of this Lemma, we have that $\mathcal{C}_{-X^{\top}}$ is not MRD and therefore the same holds for $\mathcal{C}_{X}$. Hence, by part (1) of Proposition 3 we can conclude that $\mathcal{C}_{X}$ is not MRD.

The following result derives from [17, Corollary 3.3] and it gives conditions for a code $C_{X}$ to be MRD, based only on the matrix $X$. For this purpose, we first introduce the set of normalized upper triangular matrices as

$$
\mathrm{U}_{r}(q):=\left\{A \in \mathbb{F}_{q}^{r \times r} \mid a_{i, j}=0 \text { for all } i>j, a_{i, i}=1 \text { for all } i\right\} .
$$

Theorem 8. Let $X \in \mathbb{F}_{q^{m}}^{k \times(n-k)}$. The following are equivalent:

(1) $C_{X}$ is MRD.

(2) For every $A \in \mathrm{GL}_{k}(q), B \in \mathrm{GL}_{n-k}(q), C \in \mathbb{F}_{q}^{k \times(n-k)}$, the matrix $A X B+C$ is superregular.

(3) For every $A \in \mathrm{U}_{k}(q), B \in \mathrm{U}_{n-k}(q), C \in \mathbb{F}_{q}^{k \times(n-k)}$, the matrix $A X B+C$ is superregular.

Proof. $(2) \Rightarrow(3)$ This is clear, since $\mathrm{U}_{r}(q) \subseteq \mathrm{GL}_{r}(q)$ for any positive integer $r$.

$(1) \Rightarrow(2)$ Suppose that $C_{X}$ is MRD, and let $A \in \mathrm{GL}_{k}(q), B \in \mathrm{GL}_{n-k}(q), C \in \mathbb{F}_{q}^{k \times(n-k)}$. Then we consider the matrix $\widetilde{G}=\left(I_{k} \mid X\right) M$, where

$$
M:=\left(\begin{array}{cc}
A^{-1} & A^{-1} C \\
0 & B
\end{array}\right) \in \operatorname{GL}_{n}(q) .
$$

Then it is easy to see that $\operatorname{rs}(\widetilde{G})=C_{\widetilde{X}}$, where $\widetilde{X}=A X B+C$. Then the statement follows from Proposition 4 and the characterization of MDS codes given in Theorem 2.

$(3) \Rightarrow(1)$ Suppose that 3 holds. Every matrix $M \in \mathrm{U}_{n}(q)$ can be written in the form

$$
M:=\left(\begin{array}{cc}
A & A C \\
0 & B
\end{array}\right) \in \mathrm{GL}_{n}(q),
$$


for some $A \in \mathrm{U}_{k}(q), B \in \mathrm{U}_{n-k}(q), C \in \mathbb{F}_{q}^{k \times(n-k)}$. Moreover, $\operatorname{rs}\left(\left(I_{k} \mid X\right) M\right)=C_{\tilde{X}}$, where $X=A^{-1} X B+C$. Since the map $A \longmapsto A^{-1}$ is a bijection from $\mathrm{U}_{k}(q)$ into itself, we conclude that $C_{X}$ is an MRD code using Theorem 2 and [17, Corollary 3.3].

Theorem 8 can be considered as the analogue in the rank metric of Theorem 2 and can also be found in [28, Theorem 3.11]. The equivalence between parts 1 and 3 has been shown independently in [1, Theorem 4].

\section{Standard Form of Gabidulin Codes}

Analogously to the works of Roth and Seroussi [40] and Dür [14] for GRS codes, in this section we characterize the matrices $X \in \mathbb{F}_{q^{m}}^{k \times(n-k)}$ such that the code $\mathcal{C}_{X}$ is a generalized Gabidulin code, and we refer to this family of matrices as $(q, s)$-Cauchy matrices. In order to do that, we rely on Theorem 7 which tells that $\operatorname{rk}\left(\Phi_{s}(X)\right)=1$. Therefore, we start with a rank-one matrix $A$ and determine the conditions such that $A$ belongs to the image of the map $\Phi_{s}$. Finally, we impose that the resulting matrices $X$ with $\Phi_{s}(X)=A$, are such that the code $\mathcal{C}_{X}$ is MRD and get the desired characterization.

Furthermore, we also give an analogue of Theorem 4 for generalized Gabidulin codes. This result represents a new criterion that allows to determine whether a given code in standard form is a generalized Gabidulin code, which is faster than the one given in Theorem 7 .

As in the whole work, we fix positive integers $0<k<n \leq m$. For every positive integer $s$ with $\operatorname{gcd}(m, s)=1$, we consider the following sets:

$$
\begin{aligned}
\mathbb{G}(s) & :=\left\{X \in \mathbb{F}_{q^{m}}^{k \times(n-k)} \mid \mathcal{C}_{X} \in \operatorname{Gab}_{q}(k, n, m, s)\right\}, \\
\mathcal{R}_{1}^{*} & :=\left\{A \in\left(\mathbb{F}_{q^{m}}^{*}\right)^{k \times(n-k)} \mid \operatorname{rk}(A)=1\right\}, \\
\mathcal{K} & :=\left(\operatorname{ker}\left(\operatorname{Tr}_{\mathbb{F}_{q^{m} / \mathbb{F}_{q}}}\right)\right)^{k \times(n-k)} .
\end{aligned}
$$

Lemma 8. For every integer s coprime to $m$, the following properties hold.

(1) $\Phi_{s}\left(\mathbb{F}_{q^{m}}^{k \times(n-k)}\right)=\mathcal{K}$.

(2) Let $A \in \mathbb{F}_{q^{m}}^{k \times(n-k)}$. If $A \in \mathcal{K}$ and $X \in \Phi_{s}^{-1}(\{A\})$, then

$$
\Phi_{s}^{-1}(\{A\})=\left\{X+B \mid B \in \mathbb{F}_{q}^{k \times(n-k)}\right\} .
$$

In particular,

$$
\left|\Phi_{s}^{-1}(\{A\})\right|= \begin{cases}0 & \text { if } A \notin \mathcal{K} \\ q^{k(n-k)} & \text { if } A \in \mathcal{K} .\end{cases}
$$

(3) $\Phi_{s}(\mathbb{G}(s)) \subseteq \mathcal{R}_{1}^{*} \cap \mathcal{K}$, or, equivalently, $\mathbb{G}(s) \subseteq \Phi_{s}^{-1}\left(\mathcal{R}_{1}^{*} \cap \mathcal{K}\right)$.

(4) Let $A \in \mathcal{R}_{1}^{*} \cap \mathcal{K}$ and $X \in \Phi_{s}^{-1}(\{A\})$. If $X \in \mathbb{G}(s)$ then the whole preimage of $\{A\}$ is contained in $\mathbb{G}(s)$, i.e.

$$
\Phi_{s}^{-1}(\{A\}) \subseteq \mathbb{G}(s) .
$$

Proof. (1) Since $\Phi_{s}$ is the function that maps every entry $x_{i, j}$ of the matrix $X$ to $\varphi_{s}\left(x_{i, j}\right)$, we have that $A \in \Phi_{s}\left(\mathbb{F}_{q^{m}}^{k \times(n-k)}\right)$ if and only if every entry $a_{i, j}$ of $A$ belongs to $\operatorname{Im}\left(\varphi_{s}\right)$. By part (5) of Lemma 1 this is true if and only if every $a_{i, j}$ belongs to ker $\left(\operatorname{Tr}_{\mathbb{F}_{q} / \mathbb{F}_{q}}\right)$.

(2) If $A \notin \mathcal{K}$, then, by part (1) of this Lemma, this means that $\Phi_{s}^{-1}(A)=\emptyset$. Otherwise, again by part $(1), \Phi_{s}^{-1}(A) \neq \emptyset$. In this case every entry $a_{i, j}$ belongs to $\operatorname{Im}\left(\varphi_{s}\right)$, and by part (2) of Lemma 3 ,

$$
\varphi_{s}^{-1}\left(\left\{a_{i, j}\right\}\right)=\left\{x_{i, j}+\lambda \mid \lambda \in \mathbb{F}_{q}\right\}
$$

for some $x \in \mathbb{F}_{q^{m}}$. Since this holds for every entry, we get the desired result. 
(3) Let $X \in \mathbb{G}(s)$. By Theorem $7, \Phi_{s}(X)$ has rank equal to 1 . Moreover, by Lemma 5 , all the entries of $\Phi_{s}(X)$ are in $\mathbb{F}_{q^{m}}^{*}$. Finally, by part (1) of this Lemma, we have $\Phi_{s}(X) \in \mathcal{K}$ and this concludes the proof.

(4) It directly follows from part (2) of this Lemma and part (2) of Corollary 4.

As a consequence of part (4) of Lemma 8, given a matrix $X \in \mathbb{F}_{q^{m}}^{k \times(n-k)}$, we have that the property of $\mathcal{C}_{X}$ being Gabidulin only depends on the image $\Phi_{s}(X)$. It is now crucial to investigate the matrices that belong to the image of the map $\Phi_{s}$, and, by part (3) of Lemma 8, in particular $\mathcal{R}_{1}^{*} \cap \mathcal{K}$.

By definition, every element in $\mathcal{R}_{1}^{*} \cap \mathcal{K}$ has rank one, and it is well-known that every rankone matrix can be written as the product of a non-zero column vector by a non-zero row vector. Moreover, for a fixed rank-one matrix over $\mathbb{F}_{q^{m}}$, there are exactly $q^{m}-1$ different parametrizations of this form.

The following result is straightforward and directly follows from the considerations above and the definitions of $\mathcal{R}_{1}^{*}$ and $\mathcal{K}$.

Lemma 9. The set $\mathcal{R}_{1}^{*} \cap \mathcal{K}$ can be written in the following way

$$
\begin{aligned}
\mathcal{R}_{1}^{*} \cap \mathcal{K} & =\left\{\alpha^{\top} \beta \mid \alpha \in \mathbb{F}_{q^{m}}^{k}, \beta \in \mathbb{F}_{q^{m}}^{n-k}, \alpha_{i} \beta_{j} \in \operatorname{ker}\left(\operatorname{Tr}_{\mathbb{F}_{q m} / \mathbb{F}_{q}}\right) \text { for all } i, j\right\} \\
& =\left\{\alpha^{\top} \beta \mid \alpha \in \mathbb{F}_{q^{m}}^{k}, \beta \in \mathbb{F}_{q^{m}}^{n-k}, \beta_{j} \in \operatorname{supp}_{q}(\alpha)^{\times} \text {for all } j\right\} \\
& =\left\{\alpha^{\top} \beta \mid \alpha \in \mathbb{F}_{q^{m}}^{k}, \beta \in \mathbb{F}_{q^{m}}^{n-k}, \operatorname{supp}_{q}(\beta) \operatorname{supp}_{q}(\alpha)^{\times}\right\}
\end{aligned}
$$

Moreover, every element in $\mathcal{R}_{1}^{*} \cap \mathcal{K}$ has $q^{m}-1$ distinct representations of this form.

This result gives a convenient way to represent $\mathcal{R}_{1}^{*} \cap \mathcal{K}$ using the set

$$
V_{k, n}:=\left\{(\alpha, \beta) \in \mathbb{F}_{q^{m}}^{k} \times \mathbb{F}_{q^{m}}^{n-k} \mid \operatorname{supp}_{q}(\beta) \operatorname{supp}_{q}(\alpha)^{\times}\right\} .
$$

Notice that, since we have $q^{m}-1$ distinct representations for a matrix in $\mathcal{R}_{1}^{*} \cap \mathcal{K}$ and the entries are all non-zero, we can always choose the representation with $\beta_{1}=1$.

At this point, given $(\alpha, \beta) \in V_{k, n}$ and a matrix $X \in \Phi_{s}^{-1}\left(\left\{\alpha^{\top} \beta\right\}\right)$, we have, by Theorem 7 and by the definition of $\mathbb{G}(s)$, that $\mathcal{C}_{X}$ is MRD if and only if $X \in \mathbb{G}(s)$, i.e. if and only if $\mathcal{C}_{X}$ is a generalized Gabidulin code of parameter $s$.

Lemma 10. Let $(\alpha, \beta) \in V_{k, n}$, where $\alpha=\left(\alpha_{1}, \ldots, \alpha_{k}\right)$ and $\beta=\left(\beta_{1}, \ldots, \beta_{n-k}\right)$ and let

$$
X \in \Phi_{s}^{-1}\left(\left\{\alpha^{\top} \beta\right\}\right) .
$$

(1) If $\operatorname{rk}_{q}(\alpha)<k$, then $X \notin \mathbb{G}(s)$, i.e. $\mathcal{C}_{X}$ is not $M R D$.

(2) If $\operatorname{rk}_{q}(\beta)<n-k$, then $X \notin \mathbb{G}(s)$, i.e. $\mathcal{C}_{X}$ is not $M R D$.

Proof. (1) The entries of the first column of $\alpha^{\top} \beta$ are $\alpha_{1} \beta_{1}, \ldots, \alpha_{k} \beta_{1}$ that by hypothesis are $\mathbb{F}_{q}$-linearly dependent. By Lemma 4 this means that the entries of the first column of $X$ together with the element 1 , are $\mathbb{F}_{q}$-linearly dependent. At this point we conclude by Lemma 7 .

(2) The entries of the first row of $\alpha^{\top} \beta$ are $\alpha_{1} \beta_{1} \ldots, \alpha_{1} \beta_{n-k}$ that by hypothesis are $\mathbb{F}_{q^{-}}$ linearly dependent. Then we conclude again using Lemma 4 and Lemma 7.

Finally, we can state our desired result.

Theorem 9 (Standard form of Gabidulin codes). Suppose $X \in \mathbb{F}_{q^{m}}^{k \times(n-k)}$ is a matrix such that $\mathcal{C}_{X} \in \operatorname{Gab}_{q}(k, n, m, s)$. Then $X \in \Phi_{s}^{-1}\left(\left\{\alpha^{\top} \beta\right\}\right)$ for some $\alpha \in \mathbb{F}_{q^{m}}^{k}, \beta \in \mathbb{F}_{q^{m}}^{n-k}$ such that
(a) $\operatorname{rk}_{q}(\alpha)=k$,
(b) $\operatorname{rk}_{q}(\beta)=n-k$,
(c) $\operatorname{supp}_{q}(\beta) \subseteq \operatorname{supp}_{q}(\alpha)^{\times}$. 
Moreover, if $\alpha \in \mathbb{F}_{q^{m}}^{k}, \beta \in \mathbb{F}_{q^{m}}^{n-k}$ satisfy properties (a), (b), (c) and $X \in \Phi_{s}^{-1}\left(\left\{\alpha^{\top} \beta\right\}\right)$, then $\mathcal{C}_{X} \in G a b_{q}(k, n, m, s)$.

Proof. Let $\mathcal{C}_{X}$ be a Gabidulin code. We have that $\Phi_{s}(X)$ is of the form $\alpha^{\top} \beta$ for some $\alpha, \beta$ by part (3) of Lemma 8 and by Lemma 9 . Moreover, part (c) follows from the fact that if $\mathcal{C}_{X}$ is a Gabidulin code, then all the entries of $\Phi_{s}(X)$ belong to $\operatorname{ker}\left(\operatorname{Tr}_{\mathbb{F}_{q} m / \mathbb{F}_{q}}\right)$. Finally part (a) and (b) follow from Lemma 10.

On the other hand, we can count the number of matrices $X \in \Phi_{s}^{-1}\left(\left\{\alpha^{\top} \beta\right\}\right)$ for $\alpha \in \mathbb{F}_{q^{m}}^{k}, \beta \in$ $\mathbb{F}_{q^{m}}^{n-k}$ satisfying properties (a), (b), (c). For $\alpha$ we have $\prod_{i=0}^{k-1}\left(q^{m}-q^{i}\right)$ possible choices, while for $\beta$ we have $\prod_{i=0}^{n-k-1}\left(q^{m-k}-q^{i}\right)$ choices. Moreover we need to divide by $q^{m}-1$ since, by Lemma 9, we have $q^{m}-1$ choices of $(\alpha, \beta)$ that gives the same matrix $\alpha^{\top} \beta$. Since for every $\alpha^{\top} \beta \in \mathcal{R}_{1}^{*} \cap \mathcal{K}$ we have, by part (2) of Lemma $8, q^{k(n-k)}$ many matrices in the preimage under the map $\Phi_{s}$, we finally obtain

$$
\begin{aligned}
& \frac{q^{k(n-k)}}{q^{m}-1} \prod_{i=0}^{k-1}\left(q^{m}-q^{i}\right) \prod_{i=0}^{n-k-1}\left(q^{m-k}-q^{i}\right) \\
= & \prod_{i=1}^{k-1}\left(q^{m}-q^{i}\right) \prod_{i=0}^{n-k-1}\left(q^{m}-q^{i+k}\right) \\
= & \prod_{i=1}^{n-1}\left(q^{m}-q^{i}\right) .
\end{aligned}
$$

By Corollary 3, this number is equal to the number of distinct Gabidulin codes. Therefore, by a counting argument, it follows that conditions (a), (b), (c) are also sufficient.

Theorem 9 gives a characterization of the generator matrix in standard form of a generalized Gabidulin code. In [40, 14], it was shown that there is a one-to-one correspondence between generalized Reed-Solomon (GRS) codes and generalized Cauchy (GC) matrices. In that paper, it is shown that a code in the Hamming metric whose generator matrix in standard form is $\left(I_{k} \mid X\right)$ is a GRS code if and only if $X$ is a GC matrix. Since generalized Gabidulin codes are the analogue of GRS codes for the rank metric, it becomes natural to give the definition of a $q$-analogue of Cauchy matrices according to Theorem 9 .

Let $\gamma \in \mathbb{F}_{q^{m}}$ such that $\operatorname{Tr}_{\mathbb{F}_{q} m / \mathbb{F}_{q}}(\gamma) \neq 0$ and $s$ an integer coprime to $m$. We define the function $\pi_{s}$ as

$$
\begin{aligned}
\pi_{s}: \mathbb{F}_{q^{m}} & \longrightarrow \mathbb{F}_{q^{m}} \\
\alpha & \longmapsto \frac{-1}{\operatorname{Tr}_{\mathbb{F}_{q^{m}} / \mathbb{F}_{q}}(\gamma)} \sum_{i=0}^{m-2}\left(\sigma^{i+1}(\gamma) \sum_{j=0}^{i}\left(\sigma^{j}(\alpha)\right)\right) .
\end{aligned}
$$

where $\sigma:=\theta^{s}$. Recall that, by Lemma 3, for $\alpha \in \operatorname{ker}\left(\operatorname{Tr}_{\mathbb{F}_{q} m / \mathbb{F}_{q}}\right), \pi_{s}(\alpha)$ gives one of the elements in the preimage of $\varphi_{s}$, i.e. $\varphi_{s}\left(\pi_{s}(\alpha)\right)=\alpha$ and $\pi_{s}\left(\varphi_{s}(\alpha)\right)=\alpha+\lambda$ for some $\lambda \in \mathbb{F}_{q}$. Moreover, every element in $\varphi_{s}^{-1}(\{\alpha\})$ is of the form $\pi_{s}(\alpha)+\lambda$.

Definition 14. Let $\alpha \in \mathbb{F}_{q^{m}}^{t}, \beta \in \mathbb{F}_{q^{m}}^{r}$ such that

(A) $\mathrm{rk}_{q}(\alpha)=t$

(B) $\operatorname{rk}_{q}(\beta)=r$,

(C) $\operatorname{supp}_{q}(\beta) \subseteq \operatorname{supp}_{q}(\alpha)^{\times}$.

Moreover, let $s$ be an integer coprime to $m$ and $B \in \mathbb{F}_{q}^{t \times r}$. A $t \times r(q, s)$-Cauchy matrix $C_{(q, s)}(\alpha, \beta, B)$ of parameter $s$ is a matrix of the form

$$
C_{(q, s)}(\alpha, \beta, B)=\left(\begin{array}{cccc}
\pi_{s}\left(\alpha_{1} \beta_{1}\right) & \pi_{s}\left(\alpha_{1} \beta_{2}\right) & \cdots & \pi_{s}\left(\alpha_{1} \beta_{r}\right) \\
\pi_{s}\left(\alpha_{2} \beta_{1}\right) & \pi_{s}\left(\alpha_{2} \beta_{2}\right) & \cdots & \pi_{s}\left(\alpha_{2} \beta_{r}\right) \\
\vdots & \vdots & & \vdots \\
\pi_{s}\left(\alpha_{t} \beta_{1}\right) & \pi_{s}\left(\alpha_{t} \beta_{2}\right) & \cdots & \pi_{s}\left(\alpha_{t} \beta_{r}\right)
\end{array}\right)+B .
$$


When $s=1$ we will simply call it $q$-Cauchy matrix.

Remark 2. Definition 14 directly arises from the characterization of the generator matrix in standard form of a Gabidulin code. However, one can see that $(q, s)$-Cauchy matrices introduced in this work are the $q$-analogue of GC matrices. Indeed, conditions (A), (B) and (C) represent the $q$-analogues of conditions (a), (b) and (c) of Definition 7.

With this definition, we can reformulate Theorem 9 in the following way, that puts emphasis on the correspondence between generalized Gabidulin codes and $(q, s)$-Cauchy matrices

Theorem $9^{\prime}$. Let $X \in \mathbb{F}_{q^{m}}^{k \times(n-k)}$ and let $s$ be a positive integer coprime to $m$. Then, $\mathcal{C}_{X} \in$ $\operatorname{Gab}_{q}(k, n, m, s)$ if and only if the matrix $X$ is a $(q, s)$-Cauchy matrix.

From Theorem 9 we have an immediate consequence, that relates $(q, s)$-Cauchy matrices with Moore matrices.

Corollary 5. Let $0<k<n \leq m$ be positive integers and $s$ be another integer coprime to $m$. Let $g \in \mathbb{F}_{q^{m}}^{n}$ be such that $\operatorname{rk}_{q}(g)=n$. Then the matrix

$$
M_{k, s}\left(g_{1}, \ldots, g_{k}\right)^{-1} M_{k, s}\left(g_{k+1}, \ldots, g_{n}\right)
$$

is a $(q, s)$-Cauchy matrix in $\mathbb{F}_{q^{m}}^{k \times(n-k)}$.

Moreover, if $R \in \mathbb{F}_{q^{m}}^{t \times r}$ is a $(q, s)$-Cauchy matrix, then there exists $g=\left(g_{1}, \ldots, g_{t+r}\right) \in \mathbb{F}_{q^{m}}^{t+r}$ with $\operatorname{rk}_{q}(g)=t+r$ such that

$$
R=M_{t, s}\left(g_{1}, \ldots, g_{t}\right)^{-1} M_{t, s}\left(g_{t+1}, \ldots, g_{t+r}\right) .
$$

Now, we want to determine the basis of the linearized polynomial space $\mathcal{G}_{k, s}$ that corresponds to the generator matrix in standard form. In order to do that, we introduce the following notion.

Definition 15. Let $h=\left(h_{1}, \ldots, h_{\ell}\right) \in \mathbb{F}_{q^{m}}^{\ell}$ be a vector such that $\operatorname{rk}_{q}(h)=\ell$, and let $s$ be an integer coprime to $m$. We define the polynomial $p_{h, s}$ associated to $h$ as

$$
p_{h, s}(x)=\operatorname{det}\left(M_{\ell+1, s}\left(h_{1}, \ldots, h_{\ell}, x\right)\right) .
$$

Obviously $p_{h, s}(x)$ is a linearized polynomial and in particular it belongs to $\mathcal{G}_{\ell+1, s}$. Observe that, by the properties of $s$-Moore matrices, it can be deduced that the set of roots of $p_{h, s}(x)$ in $\mathbb{F}_{q^{m}}$ is equal to the $\mathbb{F}_{q^{-}}$-subspace $\operatorname{supp}_{q}(h)$. Moreover, If $h, h^{\prime} \in \mathbb{F}_{q^{m}}^{\ell}$ are two vectors such that $\operatorname{rk}_{q}(h)=\operatorname{rk}_{q}\left(h^{\prime}\right)=\ell$ and $\operatorname{supp}_{q}(h)=\operatorname{supp}_{q}\left(h^{\prime}\right)$, then

$$
p_{h, s}(x)=\operatorname{det}(E) p_{h^{\prime}, s}(x),
$$

where $E \in \mathbb{F}_{q}^{\ell \times \ell}$ is the change-of-basis matrix from $\left\{h_{1}^{\prime}, \ldots, h_{\ell}^{\prime}\right\}$ to $\left\{h_{1}, \ldots, h_{\ell}\right\}$. Note that the polynomial associated to $h$ is a scalar multiple of a particular polynomial that is known as

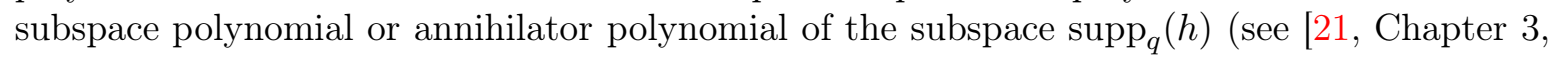
Section 4] for more details).

Remark 3. Let $\mathcal{C}=\mathcal{G}_{k, s}\left(g_{1}, \ldots, g_{n}\right)$ be a generalized Gabidulin code of parameter $s$. Consider the vectors

$$
g^{(i)}:=\left(g_{1}, \ldots, g_{i-1}, g_{i+1}, \ldots, g_{k}\right) \in \mathbb{F}_{q^{m}}^{k-1} \quad \text { for } i=1, \ldots, k,
$$

and define the polynomials

$$
f_{i}(x):=p_{g^{(i)}, s}\left(g_{i}\right)^{-1} p_{g^{(i)}, s}(x) \quad \text { for } i=1, \ldots, k .
$$

It follows from the definition that for every $i, j \in\{1, \ldots, k\}$, we have $f_{i}(x) \in \mathcal{G}_{k, s}$ and

$$
f_{i}\left(g_{j}\right)=\delta_{i, j}= \begin{cases}1 & \text { if } i=j \\ 0 & \text { if } i \neq j\end{cases}
$$

Therefore the generator matrix in standard form for the generalized Gabidulin code $\mathcal{C}$ is obtained evaluating the basis $\left\{f_{1}(x), \ldots, f_{k}(x)\right\}$ of $\mathcal{G}_{k, s}$ in the vector $g=\left(g_{1}, \ldots, g_{n}\right)$. 
4.1. A new criterion for generalized Gabidulin codes. The following result represents the analogue of Theorem 4 for the rank metric, and its proof directly follows from Theorem 9.

Theorem 10 (New Gabidulin Criterion I). Let $X \in \mathbb{F}_{q^{m}}^{k \times(n-k)}$ and let $s$ be an integer coprime to $m$. Then, $\mathcal{C}_{X} \in \operatorname{Gab}_{q}(k, n, m, s)$ if and only if

(i) the first row of the matrix $\Phi_{s}(X)$ has q-rank $n-k$

(ii) the first column of the matrix $\Phi_{s}(X)$ has q-rank $k$,

(iii) $\operatorname{rk}\left(\Phi_{s}(X)\right)=1$.

This theorem can be reformulated also in the following way.

Theorem 10' (New Gabidulin Criterion II). Let

$$
X=\left(x_{i, j}\right) \underset{\substack{1 \leq i \leq k \\ 1 \leq j \leq n-k}}{ } \in \mathbb{F}_{q^{m}}^{k \times(n-k)}
$$

and let $s$ be an integer coprime to $m$. Then, $\mathcal{C}_{X} \in \operatorname{Gab}_{q}(k, n, m, s)$ if and only if

(i') $\operatorname{rk}_{q}\left(1, x_{1,1}, \ldots, x_{1, n-k}\right)=n-k+1$,

(ii') $\operatorname{rk}_{q}\left(1, x_{1,1}, \ldots, x_{k, 1}\right)=k+1$,

(iii) $\operatorname{rk}\left(\Phi_{s}(X)\right)=1$.

Proof. By Lemma 4 we have that conditions (i') and (ii') are equivalent to conditions (i) and (ii). This means that the statement is equivalent to Theorem 10.

In addition to representing a natural analogue of Theorem 4 for the rank metric framework, Theorem 10 also gives a new criterion to recognize whether a given code in standard form is a generalized Gabidulin code. Observe that, contrary to Theorem 7, that gives a criterion subject to a previous verification that the code is MRD, this result is independent on this assumption, and it could be verified more easily. Indeed, according to Proposition 4, checking whether a code is MRD requires the computation of $\left(\begin{array}{l}n \\ k\end{array}\right)_{q}=\mathcal{O}\left(q^{k(n-k)}\right)$ matrix products and ranks, while this new criterion only requires to check the linear independence of two sets of elements and the computation of the rank of one matrix.

More generally, suppose we have an $\mathbb{F}_{q^{m}}$-linear rank metric code $\mathcal{C} \subseteq \mathbb{F}_{q^{m}}^{n}$ of dimension $k$ given by one of its generator matrices $G \in \mathbb{F}_{q^{m}}^{k \times n}$, and an integer $s$ coprime to $m$. We can check whether $\mathcal{C}$ is a generalized Gabidulin code of parameter $s$ with the following algorithm. First we compute the reduced row echelon form of $G$. If it is not of the form $\left(I_{k} \mid X\right)$, then by Lemma $5, \mathcal{C}$ is not MRD and hence it is not a generalized Gabidulin code for any parameter $s$. Hence, suppose we get a matrix of the form $\left(I_{k} \mid X\right)$. We can use Theorem 10, computing the matrix $\Phi_{s}(X)$ and its rank, and then verifying the linear independence of the elements in first row and in the first column. It is easy to see that the computational cost of this algorithm is given by the cost of computing the reduced row echelon form of $G$, that can be done via Gaussian elimination, or with faster algorithms. Therefore we have just provided a procedure that verifies if a given code is a Gabidulin code with $\mathcal{O}(m \cdot F(k, n))$ operations over $\mathbb{F}_{q^{m}}$, where $F(k, n)$ represents the computational cost of computing the reduced row echelon form of a $k \times n$ matrix. Observe that new criteria for checking whether a given rank-metric code is a generalized Gabidulin code were given in [31, Theorem 6.5]. Although these criteria do not require to check the $\mathrm{MRD}$ condition, it is easy to see that the procedure described above is still faster.

Example 1. Let $q=3, k=3$ and $n=m=6$. Consider the finite field $\mathbb{F}_{3^{6}}=\mathbb{F}_{3}(a)$, where $a$ is a primitive element that satisfies the relation $a^{6}+2 a^{4}+a^{2}+2 a+2=0$. Consider the $\mathbb{F}_{3^{6}}$-linear code $\mathcal{C} \subseteq \mathbb{F}_{3^{6}}^{6}$ with generator matrix

$$
G=\left(\begin{array}{cccccc}
a^{2} & a^{54} & a^{591} & a^{277} & a^{160} & a^{634} \\
a^{67} & a^{701} & a^{443} & a^{45} & a^{486} & a^{209} \\
a^{320} & a^{199} & a^{650} & a^{361} & a^{701} & a^{562}
\end{array}\right)
$$


We put $G$ in reduced row echelon form, and obtain the matrix $\left(I_{3} \mid X\right)$ with

$$
X=\left(\begin{array}{ccc}
a^{180} & a^{373} & a^{714} \\
a^{14} & a^{588} & a^{561} \\
a^{370} & a^{702} & a^{442}
\end{array}\right)
$$

For $s=1$ we consider the map

$$
\begin{aligned}
\pi_{1}: \mathbb{F}_{3^{6}} & \longrightarrow \mathbb{F}_{3^{6}} \\
z & \longmapsto \sum_{i=0}^{4}\left(\gamma^{3^{i+1}} \sum_{j=0}^{i} z^{3^{j}}\right),
\end{aligned}
$$

with $\gamma=a^{2}$. Then, we compute the matrix

$$
\Phi_{1}(X)=\left(\begin{array}{ccc}
a^{72} & a^{226} & a^{406} \\
a^{98} & a^{252} & a^{432} \\
a^{144} & a^{298} & a^{478}
\end{array}\right)
$$

and observe that it has rank one. Moreover, the element of the first row of $\Phi_{1}(X)$ are linearly independent over $\mathbb{F}_{3}$ and the same holds for the elements of the first column. Thus, by Theorem $10, \mathcal{C}$ is a generalized Gabidulin code of parameter 1, that is a classical Gabidulin code.

4.2. Recovering the parameters of the code from the $(q, s)$-Cauchy matrix. In order to complete the picture on the correspondence between generalized Gabidulin codes and $(q, s)$ Cauchy matrices, we need to find the relations between the vector of points $g=\left(g_{1}, \ldots, g_{n}\right)$ in which the set $\mathcal{G}_{k, s}$ is evaluated, and the corresponding vectors $\alpha=\left(\alpha_{1}, \ldots, \alpha_{k}\right) \in \mathbb{F}_{q^{m}}^{k}, \beta=$ $\left(\beta_{1}, \ldots, \beta_{n-k}\right) \in \mathbb{F}_{q^{m}}^{n-k}$ and matrix $B \in \mathbb{F}_{q}^{k \times(n-k)}$ that define the $(q, s)$-Cauchy matrix. Observe that, by Lemma 9 and Proposition 5 , we can always suppose $\beta_{1}=g_{1}=1$. In the rest of this section we will always use this assumption.

As a preliminary result, we prove that knowing the entries of a $(q, s)$-Cauchy matrix is equivalent to knowing its defining parameters $\alpha, \beta$ and $B$. If one knows the latter, then it is trivial that the entries of the $(q, s)$-Cauchy matrix can be easily computed. For the other way around we have the following result.

Proposition 6. Let $X \in \mathbb{F}_{q^{m}}^{t \times r}$ be a $(q, s)$-Cauchy matrix. Then it is possible to recover the parameters $\alpha \in \mathbb{F}_{q^{m}}^{t}, \beta \in \mathbb{F}_{q^{m}}^{r}$ and $B \in \mathbb{F}_{q}^{t \times r}$ from the entries of $X$.

Proof. It follows from the definition of $\pi_{s}$ and from Lemma 3 that $\varphi_{s}\left(x_{i, 1}\right)=\alpha_{i}\left(\right.$ since $\left.\beta_{1}=1\right)$, and $\varphi_{s}\left(x_{i, j}\right)=\alpha_{i} \beta_{j}$ for $j=2, \ldots, r$. From that, we can recover $\alpha$ and $\beta$. Finally, the matrix $B$ can be easily obtained, since

$$
B=X-\left(\begin{array}{cccc}
\pi_{s}\left(\alpha_{1} \beta_{1}\right) & \pi_{s}\left(\alpha_{1} \beta_{2}\right) & \cdots & \pi_{s}\left(\alpha_{1} \beta_{r}\right) \\
\pi_{s}\left(\alpha_{2} \beta_{1}\right) & \pi_{s}\left(\alpha_{2} \beta_{2}\right) & \cdots & \pi_{s}\left(\alpha_{2} \beta_{r}\right) \\
\vdots & \vdots & & \vdots \\
\pi_{s}\left(\alpha_{t} \beta_{1}\right) & \pi_{s}\left(\alpha_{1} \beta_{2}\right) & \cdots & \pi_{s}\left(\alpha_{t} \beta_{r}\right)
\end{array}\right)
$$

Suppose we have a generalized Gabidulin code $\mathcal{C}=\mathcal{G}_{k, s}\left(g_{1}, \ldots, g_{n}\right)$. Then we can efficiently obtain the corresponding $(q, s)$-Cauchy matrix by computing the reduced row echelon form of the $s$-Moore matrix $M_{k, s}\left(g_{1}, \ldots, g_{n}\right)$. The cost of this reduction is $\mathcal{O}(F(k, n))$ field operations over the finite field $\mathbb{F}_{q^{m}}$, where $F(k, n)$ is the cost of computing the reduced row echelon form of a $k \times n$ matrix. If we want a more explicit way to do it (but less efficient), then we can compute the basis $\left\{f_{1}(x), \ldots, f_{k}(x)\right\}$ of $\mathcal{G}_{k, s}$ as described in Remark 3 , and evaluate it in the vector $g=\left(g_{1}, \ldots, g_{n}\right)$. In order to recover the parameters $\alpha \in \mathbb{F}_{q^{m}}^{k}, \beta \in \mathbb{F}_{q^{m}}^{n-k}$ and $B \in \mathbb{F}_{q}^{k \times(n-k)}$, one can use Proposition 6.

On the other hand, we have that the two sets of parameters that we want to put in relation, are connected by Corollary 5 as follows: 


$$
M_{k, s}\left(g_{1}, \ldots, g_{k}\right) C_{(q, s)}(\alpha, \beta, B)=M_{k, s}\left(g_{k+1}, \ldots, g_{n}\right) .
$$

From this matrix equation we can deduce how to get the vector $g$ from $\alpha, \beta$ and $B$. Let $\sigma:=\theta^{s}$, where $\theta$ is the $q$-Frobenius automorphism of $\mathbb{F}_{q^{m}}$. Since $\beta_{1}=1$, from the first column of the matrix product we get

$$
\sum_{j=1}^{k} g_{j}\left(\pi_{s}\left(\alpha_{j}\right)+b_{j, 1}\right)=g_{k+1}
$$

and, in general, for $\ell=0, \ldots, k-1$,

$$
\sum_{j=1}^{k} \sigma^{\ell}\left(g_{j}\right)\left(\pi_{s}\left(\alpha_{j}\right)+b_{j, 1}\right)=\sigma^{\ell}\left(g_{k+1}\right),
$$

where we have set $\sigma=\theta^{s}$. If we apply $\sigma$ to equation (3) for $\ell-1$ and we subtract (3) to it, we get the set of equations

$$
\begin{aligned}
0 & =\sum_{j=1}^{k} \sigma^{\ell}\left(g_{j}\right)\left(\sigma\left(\pi_{s}\left(\alpha_{j}\right)+b_{j, 1}\right)-\left(\pi_{s}\left(\alpha_{j}\right)+b_{j, 1}\right)\right) \\
& =\sum_{j=1}^{k} \sigma^{\ell}\left(g_{j}\right) \alpha_{j},
\end{aligned}
$$

for every $\ell=1, \ldots, k-1$, where the last identity follows from part (2) of Lemma 3.

We can repeat this process with any other column of the matrix product, and we get, for $i=2, \ldots, n-k$,

and

$$
\sum_{j=1}^{k} g_{j}\left(\pi_{s}\left(\alpha_{j} \beta_{i}\right)+b_{j, i}\right)=g_{k+i}
$$

$$
0=\sum_{j=1}^{k} \sigma^{\ell}\left(g_{j}\right) \alpha_{j} \beta_{i} .
$$

However, this set of equations is the same as (4), therefore we do not consider it. Now, we can show that equations (2), (4) and (5) are exactly what we need for our purpose.

By Proposition 6 , we can recover the vectors $\alpha$ and $\beta$ and the matrix $B$ from $X$. Moreover, applying $\sigma^{-\ell}$ to every equation in (4), we get a linear system

$$
\left(\begin{array}{cccc}
\sigma^{-1}\left(\alpha_{2}\right) & \sigma^{-1}\left(\alpha_{3}\right) & \cdots & \sigma^{-1}\left(\alpha_{k}\right) \\
\sigma^{-2}\left(\alpha_{2}\right) & \sigma^{-2}\left(\alpha_{3}\right) & \cdots & \sigma^{-2}\left(\alpha_{k}\right) \\
\vdots & \vdots & & \vdots \\
\sigma^{-k+1}\left(\alpha_{2}\right) & \sigma^{-k+1}\left(\alpha_{3}\right) & \cdots & \sigma^{-k+1}\left(\alpha_{k}\right)
\end{array}\right)\left(\begin{array}{c}
g_{2} \\
\vdots \\
g_{k}
\end{array}\right)=\left(\begin{array}{c}
\sigma^{-1}\left(\alpha_{1}\right) \\
\sigma^{-2}\left(\alpha_{1}\right) \\
\vdots \\
\sigma^{-k+1}\left(\alpha_{1}\right)
\end{array}\right)
$$

with $g_{2}, \ldots, g_{k}$ unknowns. The matrix defining the linear system $(6)$ is a $(k-1) \times(k-1)$ matrix with coefficients in $\mathbb{F}_{q^{m}}$. In particular, this matrix is equal to the Moore matrix $M_{k-1,-s}\left(\sigma^{-1}\left(\alpha_{2}\right), \ldots, \sigma^{-1}\left(\alpha_{k}\right)\right)$, and since $\alpha_{2}, \ldots, \alpha_{k}$ are $\mathbb{F}_{q}$-linearly independent it has full rank. The unique solution of this linear system allows to compute $g_{2}, \ldots, g_{k}$, and for computing $g_{k+1}, \ldots, g_{n}$ one can use (2) and (5).

\section{Gabidulin codes in Hankel and Toeplitz Form}

In this section we use the characterization of the generator matrix in standard form for a generalized Gabidulin code given in Section 4 for the construction of particular subclasses of these codes. Indeed, we will prove that there exist generalized Gabidulin codes $\mathcal{C}_{X}$ such that $X$ is a Hankel matrix or a Toeplitz matrix.

For our purpose, we first need a technical result. 
Lemma 11. Let $\gamma \in \mathbb{F}_{q^{m}}$ be a primitive element, i.e. such that $\mathbb{F}_{q^{m}}^{*}=\langle\gamma\rangle$. Then there exists $\ell \in \mathbb{N}$ such that

$$
\operatorname{Tr}_{\mathbb{F}_{q^{m}} / \mathbb{F}_{q}}\left(\gamma^{\ell}\right)=\operatorname{Tr}_{\mathbb{F}_{q^{m}} / \mathbb{F}_{q}}\left(\gamma^{\ell+1}\right)=\ldots=\operatorname{Tr}_{\mathbb{F}_{q^{m}} / \mathbb{F}_{q}}\left(\gamma^{\ell+m-2}\right)=0 .
$$

Proof. Since $\gamma$ is a primitive element, then $\mathbb{F}_{q^{m}}=\mathbb{F}_{q}(\gamma)$ and $1, \gamma, \gamma^{2}, \ldots, \gamma^{m-1}$ is an $\mathbb{F}_{q^{-} \text {-basis }}$ of $\mathbb{F}_{q^{m}}$. Consider the $\mathbb{F}_{q^{-}}$linear map $L \in \operatorname{Hom}_{\mathbb{F}_{q}}\left(\mathbb{F}_{q^{m}}, \mathbb{F}_{q}\right)$ defined as

$$
L\left(\gamma^{i}\right)= \begin{cases}0 & \text { for } 0 \leq i \leq m-2 \\ 1 & \text { for } i=m-1 .\end{cases}
$$

$L$ is a non zero element in $\operatorname{Hom}_{\mathbb{F}_{q}}\left(\mathbb{F}_{q^{m}}, \mathbb{F}_{q}\right)$, and by Theorem 1 , there exists $\beta \in \mathbb{F}_{q^{m}}^{*}$ such that $L=T_{\beta}$. At this point, since $\gamma$ is a primitive element, there exists $\ell \in \mathbb{N}$ such that $\beta=\gamma^{\ell}$. In this way, we have that for all $i=0, \ldots, m-2$,

$$
\operatorname{Tr}_{\mathbb{F}_{q} m / \mathbb{F}_{q}}\left(\gamma^{\ell+i}\right)=T_{\gamma^{\ell}}\left(\gamma^{i}\right)=L\left(\gamma^{i}\right)=0
$$

and this concludes the proof.

Definition 16. An $r \times s$ matrix $A=\left(A_{i, j}\right)$ over a field $\mathbb{F}$ is called Toeplitz matrix if there exist a vector $a=\left(a_{1-r}, a_{2-r}, \ldots, a_{s-1}\right) \in \mathbb{F}^{r+s-1}$ such that

$$
A_{i, j}=a_{j-i} \text {. }
$$

An $r \times s$ matrix $A=\left(A_{i, j}\right)$ over a field $\mathbb{F}$ is called Hankel matrix if there exist a vector $a=\left(a_{0}, a_{1}, \ldots, a_{r+s-2}\right) \in \mathbb{F}^{r+s-1}$ such that

$$
A_{i, j}=a_{i+j-2} .
$$

A special kind of square Toeplitz matrices is given by circulant matrices.

Definition 17. An $r \times r$ matrix $A=\left(A_{i, j}\right)$ over a field $\mathbb{F}$ is called circulant matrix if there exists a vector $a=\left(a_{0}, \ldots, a_{r-1}\right)$ such that

$$
A_{i, j}=a_{j-i(\bmod r)} .
$$

Theorem 11. For every $0<k<n \leq m$ and every $s$ coprime to $m$, there exists a code $\mathcal{C}_{X} \in \operatorname{Gab}_{q}(k, n, m, s)$ such that $X$ is a Hankel matrix.

Proof. Let $\gamma \in \mathbb{F}_{q^{m}}$ be a primitive element. By Lemma 11 there exist $\ell \in \mathbb{N}$ such that

$$
\operatorname{Tr}_{\mathbb{F}_{q} m / \mathbb{F}_{q}}\left(\gamma^{\ell}\right)=\operatorname{Tr}_{\mathbb{F}_{q^{m}} / \mathbb{F}_{q}}\left(\gamma^{\ell+1}\right)=\ldots=\operatorname{Tr}_{\mathbb{F}_{q^{m}} / \mathbb{F}_{q}}\left(\gamma^{\ell+m-2}\right)=0 .
$$

Let $\alpha \in \mathbb{F}_{q^{m}}^{k}, \beta \in \mathbb{F}_{q^{m}}^{n-k}$ be defined as

$$
\begin{aligned}
\alpha & =\left(\gamma^{\ell}, \gamma^{\ell+1}, \ldots, \gamma^{\ell+k-1}\right), \\
\beta & =\left(1, \gamma, \ldots, \gamma^{n-k-1}\right),
\end{aligned}
$$

and consider the matrix $\alpha^{\top} \beta$. We now check that $\alpha, \beta$ satisfy properties (a), (b), (c) of Theorem 9. Indeed, $\gamma$ is a primitive element, and therefore $1, \gamma, \ldots, \gamma^{m-1}$ are linearly independent, as well as $\gamma^{\ell}, \ldots, \gamma^{\ell+m-1}$. In particular, properties (a) and (b) are satisfied. Moreover, for every $i=0, \ldots, k-1, j=0, \ldots, n-k-1$,

$$
T_{\gamma^{\ell+i}}\left(\gamma^{j}\right)=\operatorname{Tr}_{\mathbb{F}_{q} m / \mathbb{F}_{q}}\left(\gamma^{\ell+i+j}\right)=0
$$

where the last inequality holds by (7). Therefore also property (c) is verified.

Now we have that every matrix $X \in \Phi_{s}^{-1}\left(\left\{\alpha^{\top} \beta\right\}\right)$ is a $(q, s)$-Cauchy matrix and hence it is of the form

$$
X=\left(\begin{array}{cccc}
\pi_{s}\left(\gamma^{\ell}\right) & \pi_{s}\left(\gamma^{\ell+1}\right) & \cdots & \pi_{s}\left(\gamma^{\ell+n-k-1}\right) \\
\pi_{s}\left(\gamma^{\ell+1}\right) & \pi_{s}\left(\gamma^{\ell+2}\right) & \cdots & \pi_{s}\left(\gamma^{\ell+n-k}\right) \\
\vdots & \vdots & & \vdots \\
\pi_{s}\left(\gamma^{\ell+k-1}\right) & \pi_{s}\left(\gamma^{\ell+k}\right) & \cdots & \pi_{s}\left(\gamma^{\ell+n-2}\right)
\end{array}\right)+B
$$

for an arbitrary $B \in \mathbb{F}_{q}^{k \times(n-k)}$. Choosing $B$ as a Hankel matrix completes the proof. 
Theorem 12. For every $0<k<n \leq m$ and every $s$ coprime to $m$, there exists a generalized Gabidulin code $\mathcal{C}_{X}$ of parameter $s$ such that $X$ is a Toeplitz matrix.

Proof. Following the same proof of Theorem 11 with

$$
\begin{aligned}
\alpha & =\left(\gamma^{\ell+n-k-1}, \gamma^{\ell+n-k}, \ldots, \gamma^{\ell+n-2}\right), \\
\beta & =\left(1, \gamma^{-1}, \gamma^{-2}, \ldots, \gamma^{-n+k+1}\right),
\end{aligned}
$$

the matrix obtained is of the form

$$
X=\left(\begin{array}{cccc}
\pi_{s}\left(\gamma^{\ell+n-k-1}\right) & \pi_{s}\left(\gamma^{\ell+n-k-2}\right) & \cdots & \pi_{s}\left(\gamma^{\ell}\right) \\
\pi_{s}\left(\gamma^{\ell+n-k}\right) & \pi_{s}\left(\gamma^{\ell+n-k-1}\right) & \cdots & \pi_{s}\left(\gamma^{\ell+1}\right) \\
\vdots & \vdots & & \vdots \\
\pi_{s}\left(\gamma^{\ell+n-2}\right) & \pi_{s}\left(\gamma^{\ell+n-3}\right) & \cdots & \pi_{s}\left(\gamma^{\ell+k-1}\right)
\end{array}\right)+B
$$

for an arbitrary $B \in \mathbb{F}_{q}^{k \times(n-k)}$. As above, choosing $B$ in Toeplitz form concludes the proof.

These two theorems allow to define two subfamilies of generalized Gabidulin codes, the Hankel Gabidulin codes and the Toeplitz Gabidulin codes. In the following Lemma we can see that this structure on the generator matrix in standard form is hard to improve if we still require the code to be MRD.

Lemma 12. Suppose that $n$ is even and $k=\frac{n}{2}$. Let $X \in \mathbb{F}_{q^{m}}^{k \times k}$ be a circulant matrix, and let $d$ be the minimum rank distance of the code $\mathcal{C}_{X}$. Then $d \leq 2$.

In particular, for $n \geq 4$, there does not exist any $\frac{n}{2}$-dimensional MRD code $\mathcal{C}_{X}$ with $X$ circulant matrix.

Proof. Since the matrix $X$ is circulant, then the sum of the elements on each of its columns is constant. Let $\gamma$ be such a sum. Then, the non-zero codeword of the code $\mathcal{C}_{X}$

$$
(1, \ldots, 1)\left(I_{k} \mid X\right)=(1, \ldots, 1, \gamma, \ldots, \gamma)
$$

has rank weight at most 2 . In particular, if $n \geq 4$ we have

$$
n-k+1=\frac{n}{2}+1>2 \geq d
$$

and therefore, the code $\mathcal{C}_{X}$ can not be MRD.

This result possibly suggests that, at least in the case $k=\frac{n}{2}$, it is very difficult to require more structure on the non-systematic part of the generator matrix in standard form of an MRD code. However, it would be very interesting to find new families of Gabidulin, or more generally MRD codes with structured generator matrices.

We conclude this section with a small example.

Example 2. Consider the case $q=2, k=3, n=m=6$ and $s=1$. We construct a Hankel Gabidulin code of dimension 3 and length 6 over the finite field $\mathbb{F}_{2^{6}}=\mathbb{F}_{2}(a)$, where $a$ is a primitive element that satisfies $a^{6}+a^{4}+a^{3}+a+1=0$. One can find, by Lemma 11, five consecutive powers of $a$ that belong to $\operatorname{ker}\left(\operatorname{Tr}_{\mathbb{F}_{2} / \mathbb{F}_{2}}\right)$, that are $a^{i}$ for $i=14,15, \ldots, 18$. Then, we set the vectors

$$
\alpha=\left(a^{14}, a^{15}, a^{16}\right), \quad \beta=\left(1, a, a^{2}\right) .
$$

Moreover, we choose the matrix $B$ to be the zero matrix, and the map

$$
\begin{aligned}
\pi_{1}: \mathbb{F}_{2^{6}} & \longrightarrow \mathbb{F}_{2^{6}} \\
z & \longmapsto \sum_{i=0}^{4}\left(\gamma^{2^{i+1}} \sum_{j=0}^{i} z^{2^{j}}\right),
\end{aligned}
$$

with $\gamma=a^{3}$. We then get the following $q$-Cauchy matrix

$$
X:=C_{(q, 1)}(\alpha, \beta, 0)=\left(\begin{array}{lll}
\pi_{1}\left(a^{14}\right) & \pi_{1}\left(a^{15}\right) & \pi_{1}\left(a^{16}\right) \\
\pi_{1}\left(a^{15}\right) & \pi_{1}\left(a^{16}\right) & \pi_{1}\left(a^{17}\right) \\
\pi_{1}\left(a^{16}\right) & \pi_{1}\left(a^{17}\right) & \pi_{1}\left(a^{18}\right)
\end{array}\right)=\left(\begin{array}{ccc}
a^{57} & a^{7} & a^{13} \\
a^{7} & a^{13} & a^{37} \\
a^{13} & a^{37} & a^{36}
\end{array}\right) .
$$


By Theorem 11 the code $\mathcal{C}_{X}$ is a Gabidulin code of parameter $s=1$. Moreover we can recover the vector of evaluation points $g=\left(g_{1}, \ldots, g_{6}\right)$ of the code. We can suppose $g_{1}=1$, and recover $g_{2}=a^{45}$ and $g_{3}=a^{! 5}$ using the linear system (6). Finally, using equations (2) and (5) we get $g_{4}=a^{46}, g_{5}=a^{14}$ and $g_{6}=a^{28}$. Therefore, our Hankel Gabidulin code is

$$
\mathcal{C}_{X}=\mathcal{G}_{3,1}\left(1, a^{45}, a^{15}, a^{46}, a^{14}, a^{28}\right) .
$$

\section{Conclusions and open PRoblems}

In this work we find a parametrization of the generator matrix in standard form of generalized Gabidulin codes (see Theorem 9). Such a parametrization coincides with the $q$-analogue of generalized Cauchy matrices and, therefore, leads to a natural definition of $(q, s)$-Cauchy matrices, a notion that was missing in the literature. In Theorem $9^{\prime}$ we underline that these matrices are in one-to-one correspondence with generalized Gabidulin codes. Moreover, in Theorems 10 and $10^{\prime}$ we give a new criterion for determining whether a given rank metric code of dimension $k$ and length $n$ is a generalized Gabidulin code. This new result only requires $\mathcal{O}(m \cdot F(k, n))$ field operations, where $F(k, n)$ denotes the cost of computing the reduced row echelon form of a $k \times n$ matrix, and it improves the existing criterion that relies on an a priori check of the MRD property. Finally we use our results in order to build two new subfamilies of generalized Gabidulin codes, namely the Hankel and Toeplitz Gabidulin codes (see Theorems 11 and 12). These families have the advantage that the non-systematic part of the generator matrix is a structured matrix. This implies that matrix/vector multiplications, and therefore the encoding procedure, can be performed faster than usual.

From a theoretical point of view we believe that the same parametrization as the one of Theorems 9 and $9^{\prime}$ applies to Gabidulin codes over any finite cyclic field extension $\mathbb{E} / \mathbb{K}$, which were introduced by Augot, Loidreau and Robert in [3, 2, 4] (see also [38, Section VI]). As a consequence one would also get the characterization of generalized Gabidulin codes of Theorems 10 and $10^{\prime}$ in this setting. This general approach based on general cyclic extension field is used to describe some results of this paper in [28, Chapter 4]. Here, $\sigma$-Gabidulin codes are defined with respect to a generator $\sigma$ of the Galois group $G:=\operatorname{Gal}(\mathbb{E} / \mathbb{K})$, and the space $\mathcal{G}_{k, s}$ is replaced by the space $\left\langle\mathrm{id}, \sigma, \ldots, \sigma^{k-1}\right\rangle_{\mathbb{E}} \subseteq \mathbb{E}[G]$. Formally we have the following open problem.

Problem 1. Let $\mathbb{E} / \mathbb{K}$ be an extension of fields of finite degree, and let $\mathrm{Gal}(\mathbb{E} / \mathbb{K})$ be a cyclic group. Do Theorems 9 and 9 hold in this more general setting?

Unfortunately, the proof technique used here relies on a counting argument, and therefore it does not apply to infinite fields. In order to prove the same theorems in this more general setting one needs to find a different argument, finding an explicit bijection between generalized Gabidulin codes and $(q, s)$-Cauchy matrices which also works for infinite fields.

Moreover, for future research we plan to investigate the use of this parametrization for applications in erasure and syndrome decoding for generalized Gabidulin codes.

\section{ACKNOWLEDGEMENT}

The author would like to thank Eimear Byrne for useful comments on the structure of the work and Martino Borello for suggesting to point out Lemma 12.

\section{REFERENCES}

[1] P. Almeida, U. Martínez-Peñas, and D. Napp. Systematic maximum sum rank codes. arXiv preprint arXiv:2001.07198, 2020.

[2] D. Augot. Generalization of Gabidulin codes over fields of rational functions. In 21st International Symposium on Mathematical Theory of Networks and Systems (MTNS 2014), 2014.

[3] D. Augot, P. Loidreau, and G. Robert. Rank metric and Gabidulin codes in characteristic zero. In 2013 IEEE International Symposium on Information Theory, pages 509-513. IEEE, 2013.

[4] D. Augot, P. Loidreau, and G. Robert. Generalized Gabidulin codes over fields of any characteristic. Designs, Codes and Cryptography, 86(8):1807-1848, 2018. 
[5] D. Bartoli, C. Zanella, and F. Zullo. A new family of maximum scattered linear sets in PG(1, $\left.q^{6}\right)$. arXiv preprint arXiv:1910.02278, 2019.

[6] T. P. Berger. Isometries for rank distance and permutation group of Gabidulin codes. IEEE Transactions on Information Theory, 49(11):3016 - 3019, 2003.

[7] G. M. Bergman. Ranks of tensors and change of base field. Journal of Algebra, 11(4):613-621, 1969.

[8] E. Byrne and A. Ravagnani. Partition-balanced families of codes and asymptotic enumeration in coding theory. Journal of Combinatorial Theory, Series A, 171, 2020.

[9] G. Calis and O. O. Koyluoglu. A general construction for PMDS codes. IEEE Communications Letters, 21(3):452-455, 2017.

[10] B. Csajbók, G. Marino, O. Polverino, and C. Zanella. A new family of MRD-codes. Linear Algebra and its Applications, 548:203-220, 2018.

[11] B. Csajbók, G. Marino, O. Polverino, and Y. Zhou. Maximum rank-distance codes with maximum left and right idealisers. arXiv preprint arXiv:180\%.08\%74, 2018.

[12] B. Csajbók, G. Marino, and F. Zullo. New maximum scattered linear sets of the projective line. Finite Fields and Their Applications, 54:133-150, 2018.

[13] P. Delsarte. Bilinear forms over a finite field, with applications to coding theory. Journal of Combinatorial Theory, Series A, 25(3):226-241, 1978.

[14] A. Dür. The automorphism groups of Reed-Solomon codes. Journal of Combinatorial Theory, Series A, 44(1):69-82, 1987.

[15] E. M. Gabidulin. Theory of codes with maximum rank distance. Problemy Peredachi Informatsii, 21(1):3$16,1985$.

[16] V. Guruswami and M. Sudan. Improved decoding of Reed-Solomon and algebraic-geometric codes. In Foundations of Computer Science, 1998. Proceedings. 39th Annual Symposium on, pages 28-37, 1998.

[17] A.-L. Horlemann-Trautmann and K. Marshall. New criteria for MRD and Gabidulin codes and some rank-metric code constructions. Advances in Mathematics of Communications, 11(3):533-548, 2017.

[18] R. Kötter and F. R. Kschischang. Coding for errors and erasures in random network coding. IEEE Transactions on Information Theory, 54(8):3579-3591, 2008.

[19] R. Kötter and A. Vardy. Algebraic soft-decision decoding of Reed-Solomon codes. IEEE Transactions on Information Theory, 49(11):2809-2825, 2003.

[20] A. Kshevetskiy and E. Gabidulin. The new construction of rank codes. In Information Theory, 2005. ISIT 2005. Proceedings. International Symposium on, pages 2105-2108. IEEE, 2005.

[21] R. Lidl and H. Niederreiter. Introduction to Finite Fields and their Applications. Cambridge University Press, Cambridge, London, 1994. Revised edition.

[22] P. Loidreau. Designing a rank metric based McEliece cryptosystem. In International Workshop on PostQuantum Cryptography, pages 142-152. Springer, 2010.

[23] G. Lunardon, R. Trombetti, and Y. Zhou. Generalized twisted Gabidulin codes. Journal of Combinatorial Theory, Series A, 159:79-106, 2018.

[24] P. Lusina, E. Gabidulin, and M. Bossert. Maximum rank distance codes as space-time codes. IEEE Transactions on Information Theory, 49(10):2757-2760, 2003.

[25] G. Marino, M. Montanucci, and F. Zullo. MRD-codes arising from the trinomial $x^{q}+x^{q^{3}}+c x^{q^{5}} \in \mathbb{F}_{q^{6}}[x]$. arXiv preprint arXiv:190\%.08122, 2019.

[26] K. Morrison. Equivalence for rank-metric and matrix codes and automorphism groups of Gabidulin codes. IEEE Transactions on Information Theory, 60(11):7035-7046, 2014.

[27] S. Müelich, S. Puchinger, and M. Bossert. Low-rank matrix recovery using Gabidulin codes in characteristic zero. Electronic Notes in Discrete Mathematics, 57:161-166, 2017.

[28] A. Neri. Algebraic Theory of Rank-Metric Codes: Representations, Invariants and Density Results. $\mathrm{PhD}$ thesis, Universität Zürich, 2019.

[29] A. Neri and A.-L. Horlemann-Trautmann. Random construction of Partial MDS codes. Designs, Codes and Cryptography, 2019.

[30] A. Neri, A.-L. Horlemann-Trautmann, T. Randrianarisoa, and J. Rosenthal. On the genericity of maximum rank distance and Gabidulin codes. Designs, Codes and Cryptography, 86(2):341-363, 2018.

[31] A. Neri, S. Puchinger, and A.-L. Horlemann-Trautmann. Equivalence and characterizations of linear rankmetric codes based on invariants. arXiv preprint arXiv:1911.13059, 2019.

[32] A. Neri, J. Rosenthal, and D. Schipani. Fuzzy authentication using rank distance. In International Workshop on Communication Security, pages 97-108. Springer, 2017.

[33] K. Otal and F. Özbudak. Additive rank metric codes. IEEE Transactions on Information Theory, 63(1):164-168, 2017.

[34] R. Overbeck. Structural attacks for public key cryptosystems based on Gabidulin codes. Journal of Cryptology, 21(2):280-301, 2008.

[35] S. Puchinger, J. Rosenkilde né Nielsen, and J. Sheekey. Further generalisations of twisted Gabidulin codes. In International Workshop on Coding and Cryptography (WCC), 2017. 
[36] I. S. Reed and G. Solomon. Polynomial codes over certain finite fields. Journal of the society for industrial and applied mathematics, 8(2):300-304, 1960.

[37] R. M. Roth. Maximum-rank array codes and their application to crisscross error correction. IEEE Transactions on Information Theory, 37(2):328-336, 1991.

[38] R. M. Roth. Tensor codes for the rank metric. IEEE Transactions on Information Theory, 42(6):2146-2157, 1996.

[39] R. M. Roth and A. Lempel. On MDS codes via Cauchy matrices. IEEE Transactions on Information Theory, 35(6):1314-1319, 1989.

[40] R. M. Roth and G. Seroussi. On generator matrices of MDS codes (corresp.). IEEE Transactions on Information Theory, 31(6):826-830, 1985.

[41] J. Sheekey. A new family of linear maximum rank distance codes. Advances in Mathematics of Communications, 10(3):475-488, 2016.

[42] J. Sheekey. New semifields and new MRD codes from skew polynomial rings. Journal of the London Mathematical Society, 2019.

[43] N. Silberstein, A. S. Rawat, and S. Vishwanath. Error-correcting regenerating and locally repairable codes via rank-metric codes. IEEE Transactions on Information Theory, 61(11):5765-5778, 2015.

[44] D. Silva and F. R. Kschischang. Fast encoding and decoding of Gabidulin codes. In Information Theory, 2009. ISIT 2009. IEEE International Symposium on, pages 2858-2862. IEEE, 2009.

[45] D. Silva, F. R. Kschischang, and R. Kötter. A rank-metric approach to error control in random network coding. IEEE Transactions on Information Theory, 54(9):3951 -3967, 2008.

[46] R. Singleton. Maximum distance q-nary codes. IEEE Transactions on Information Theory, 10(2):116-118, 1964.

[47] V. Tarokh, N. Seshadri, and A. R. Calderbank. Space-time codes for high data rate wireless communication: Performance criterion and code construction. IEEE transactions on information theory, 44(2):744-765, 1998.

[48] A. Wachter-Zeh, V. Afanassiev, and V. Sidorenko. Fast decoding of Gabidulin codes. Designs, codes and cryptography, 66(1-3):57-73, 2013.

[49] Z.-X. Wan. Geometry of matrices. World Scientific, Singapore, 1996. In memory of Professor L.K. Hua $(1910-1985)$. 